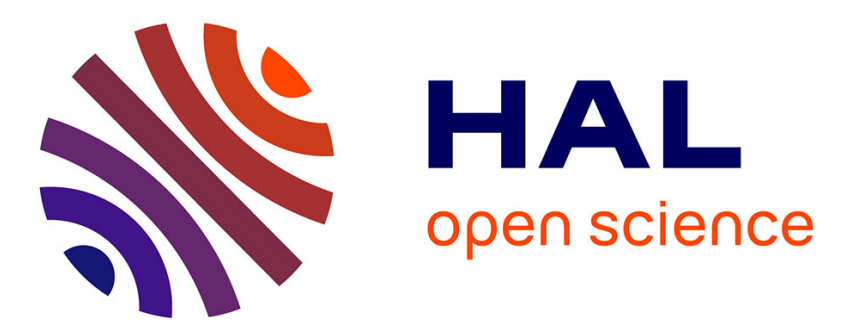

\title{
Validation and modi cation of asymptotic analysis of slow and rapid droplet spreading by numerical simulation
}

\author{
Yi Sui, Peter D.M. Spelt
}

\section{- To cite this version:}

Yi Sui, Peter D.M. Spelt. Validation and modi cation of asymptotic analysis of slow and rapid droplet spreading by numerical simulation. Journal of Fluid Mechanics, 2013, pp.283-313. hal-00742532

\section{HAL Id: hal-00742532 \\ https://hal.science/hal-00742532}

Submitted on 16 Oct 2012

HAL is a multi-disciplinary open access archive for the deposit and dissemination of scientific research documents, whether they are published or not. The documents may come from teaching and research institutions in France or abroad, or from public or private research centers.
L'archive ouverte pluridisciplinaire HAL, est destinée au dépôt et à la diffusion de documents scientifiques de niveau recherche, publiés ou non, émanant des établissements d'enseignement et de recherche français ou étrangers, des laboratoires publics ou privés. 


\title{
Validation and modification of asymptotic analysis of slow and rapid droplet spreading by numerical simulation
}

\author{
By Yi Sui ${ }^{1,2}$ and Peter D. M. Spelt ${ }^{3,4}$ \\ ${ }^{1}$ Department of Chemical Engineering, Imperial College London, London SW7 2AZ, UK \\ ${ }^{2}$ School of Engineering and Materials Science, Queen Mary University of London, London E1 \\ $4 \mathrm{NS}, \mathrm{UK}$ \\ ${ }^{3}$ Laboratoire de la Mécanique des Fluides \& d'Acoustique, CNRS, Ecully, France \\ ${ }^{4}$ Département Mécanique, Université Claude Bernard Lyon 1, Villeurbanne, France
}

(Received 15 October 2012)

Using a slip-length based level-set approach with adaptive mesh refinement, we have simulated axisymmetric droplet spreading for a dimensionless slip length down to $\mathrm{O}\left(10^{-4}\right)$. The main purpose is to validate - and where necessary improve - the asymptotic analysis of Cox (1998) for rapid droplet spreading/dewetting, in terms of the detailed interface shape in various regions close to the moving contact line and the relation between the apparent angle and the capillary number based on the instantaneous contact line speed, $\mathrm{Ca}$. Before presenting results for inertial spreading, simulation results are compared in detail with the theory of Hocking \& Rivers (1982) for slow spreading, showing these to agree very well (and in detail) for such small slip length values, although limitations in the theoretically predicted interface shape are identified; a simple extension of the theory to viscous exterior fluids is also proposed and shown to yield similar excellent agreement. For rapid droplet spreading, it is found that, in principle, the theory of Cox (1998) can predict accurately the interface shapes in the intermediate viscous sublayer, although the inviscid sublayer can only be well presented when capillary-type waves are outside the contact line region. However, $\mathrm{O}(1)$ parameters taken to be unity in Cox (1998) must be specified and terms be corrected to $\mathrm{Ca}^{+1}$ in order to achieve good agreement between the theory and the simulation, both of which are undertaken here. We also find that the apparent angle from numerical simulation, obtained by extrapolating the interface shape from the macro region to the contact line, agrees reasonably well with the modified theory of Cox (1998). A simplified version of the inertial theory is proposed in the limit of negligible viscosity of the external fluid. Building on these results, we investigate the flow structure near the contact line, the shear stress and pressure along the wall, and the use of the analysis for droplet impact and rapid dewetting. Finally, we compare the modified theory of Cox (1998) with a recent experiment for rapid droplet spreading, the results of which suggest a spreading-velocity-dependent dynamic contact angle in the experiments. The paper is closed with a discussion of the outlook regarding the potential of using the present results in large-scale simulations wherein the contact-line region is not resolved down to the slip length, especially for inertial spreading.

\section{Introduction}

We consider here the modelling of axisymmetric rapid droplet spreading, as well as rapid dewetting. Rapid contact-line motion can be distinguished from slow spreading 
through the value of an Ohnesorge number, defined here as $O h=\mu / \sqrt{\rho \sigma D}$, where $\mu$ and $\rho$ are the liquid dynamic viscosity and density, $\sigma$ is the coefficient of surface tension, and $D$ is the equivalent drop diameter. At values of $O h$ that are not very small (typically, $O h>0.01$ but this also depends on the value of the contact angle), the expected flow behaviour is gradual spreading, whereas at $O h \ll 1$ in rapid spreading capillary waves travel between the contact line and the top of the drop (above the drop centre) that may even lead to the ejection of satellite droplets (e.g., Ding et al. (2012)).

Analysis of flows with a moving contact line have revealed non-integrable singularities in shear stress and pressure at the contact line when using a no-slip condition (e.g., Moffatt (1964), Huh \& Scriven (1971), following earlier work by Taylor (1962) who had identified such singularities in scraper flows). In order to remove these, the velocity field must be made continuous at the contact line (Dussan V. \& Davis (1974)). Various mechanisms/models have been proposed that would alleviate these singularities (for a recent review see Bonn et al. (2009)). These include the widely-used approach of relaxing the no-slip condition: a slip velocity proportional to the shear rate eliminates the singularity in the shear stress although it only reduces that in the pressure to an integrable (logarithmic) singularity (Huh \& Mason (1977)); the remaining weak singularity in pressure can be suppressed by using a different slip law (e.g., replacing the slip length by the ratio of another length scale squared divided by the local height of the interface, see Sibley et al. (2012)), although this is limited to contact angles below $90^{\circ}$. Other mechanisms $/$ models include assuming the existence of a thin precursor film and accounting for intermolecular forces (although usually for small contact angles, de Gennes (1986)); a diffuse interface (Jacqmin (2000)), possibly with intermolecular forces (Pismen \& Pomeau (2000)); and surface tension relaxation (Shikhmurzaev (1993), Sibley et al. (2012)). Predictions of some of these models have been compared against each other in various studies (Savva \& Kalliadasis (2011), Sibley et al. (2012), Ding \& Spelt (2007a)). On the whole, good agreement is observed, and the small length scales in the various models, although of different origin, are usually of the same order when the predicted spreading rates agree. In the present study, we shall adopt the classical slip model, supplemented with a comparison with results obtained with a diffuse-interface model (in principle, the computational method developed herein can be extended to include a precursor film with long-range molecular forces, along the lines set out for droplet coalescence simulations by Jiang \& James (2007)). Different wetting conditions - a fixed static microscale contact angle versus a velocity dependent dynamic angle - will also be considered.

It then remains to solve the resulting governing equations for the spreading of droplets. For slow spreading, this has been pioneered by Greenspan (1978), who developed a lubrication theory, and by Hocking \& Rivers (1982), who developed a matched-asymptotic expansion. In rapid spreading, the difficulties posed by the coupled fluid flow/interface shape problem are added to by inertial effects that interfere with the contact-line region. Inertial effects are expected to play a role at a dimensionless distance $d \sim R e^{-1}$ from the contact line, where $R e$ is the Reynolds number based on the contact-line speed (all lengths herein are made dimensionless with the equivalent drop diameter $D$ ). This distance is normally much larger than the dimensions of an inner contact-line region (the dimensionless slip length $\lambda$ when using a slip model), but approaches based on matchedasymptotic expansion usually also involve a layer adjacent to such inner region of length scale $O\left(1 / \ln \left(\lambda^{-1}\right)\right)$ (e.g., Cox $\left.(1986)\right)$, and inertial effects can easily play a role on that scale. A classical analysis of contact-line motion with inertial effects along these lines has been developed by Cox (1998). Although the analysis seems valid quite generally, we undertake here a detailed validation study of this theory for rapid droplet spreading and dewetting in the regime $1 \ll R e \ll \lambda^{-1}$, not only of an apparent angle versus instanta- 
neous contact-line speed, but also of the interface shape. We also revisit the analysis of Hocking \& Rivers (1982) for slowly spreading drops.

The motivation for this study is three-fold. First, the main result of the analysis is a relation for an apparent contact angle as a function of the instantaneous contact-line speed. Formally the apparent angle is defined in asymptotic analysis of slow spreading as the angle resulting from extrapolating the interface shape on the scale of the droplet (the outer region in the asymptotic expansion) to the contact line (e.g., Hocking \& Rivers (1982), Cox (1986)). Although this does not correspond to an angle that can be measured directly anywhere along the interface, also not in slow spreading (as is demonstrated in Sec. 3), it can be determined if a large part of the interface shape is known. However, in the pertinent analysis in Cox (1998) for rapid contact-line motion, the apparent angle is merely defined as the angle in an extended intermediate region at a distance corresponding to the macroscopic length scale to the contact line. The precise definition of this angle must be adhered to when comparing with an experiment, and it seems unclear how to express one definition in terms of the other. A second motivation for this study is related to the first: matching in the analysis between various regions is achieved at points that merely follow from a scaling argument. Although this (and the definition in apparent angle) may seem to hardly matter because the slip length is very small, we conclude from our results that this is not generally correct for realistic values of a slip length for millimeter-sized drops, and any uncertainty in the analysis further adds to experimental margins of error when comparing with experiment. Finally, Cox (1998) assumed quasi-steady motion of the contact line, which is significantly challenged in rapid droplet spreading given the presence of capillary waves that interfere with the contact-line region.

More broadly, the subject of this work is to understand the contact-line motion under the complex circumstances due to inertial effects. In an earlier computational study, Ding \& Spelt (2007a) found for large slip length values that an apparent contact angle was no longer a unique function of $C a$ as a result, which is not predicted theoretically. More recently, the experiments of Ding et al. (2012) have confirmed the oscillatory motion reported in Ding \& Spelt (2007a), but also shown that the non-uniqueness vanishes. Although this seems to support the use of e.g. the analysis of Cox (1998), the apparent angle is found to exceed that predicted by Cox (1986) for any reasonable value of a slip coefficient, and the results of the model of Cox (1998) are in fact even below those of Cox (1986), making the comparison worse. It has been argued by Bayer \& Megaridis (2006), amongst others, that the microscale contact angle is actually velocity dependent. Before resorting to this argument, however, it is necessary to first confirm the analysis of Cox (1998).

High-resolution numerical simulations (using a continuum description) beyond lubrication theory have been achieved previously for other flows with moving contact lines (typically using a finite-element technique to resolve the flow in one fluid only, Lowndes (1980) for creeping flows, and Christodoulou \& Scriven (1992) for unsteady coating-type problems with inertia). In the subsequent literature, two main further approaches can be identified: simulations of droplet spreading wherein the flow is resolved down to the inner region, of the order of the slip length or equivalent length scale (e.g., Renardy et al. (2001)), and those wherein this is not resolved and instead a macroscale angle is imposed (e.g., Fukai et al. (1995), Bussmann et al. (1999)). For the present purposes, which includes resolving the flow in both fluids down to the inner region, we focus on the former category. These methods are of course limited by computational resources to rather large slip length values (typically only $\lambda=O(0.01)$ is achieved in prior work, see, e.g., Ding \& Spelt (2007a)), in contrast with the small values of the slip length re- 
quired for the asymptotic analysis to apply. In practice, the dimensionless slip length $\lambda$ is inferred to be at most $O\left(10^{-4}\right)$ for slow spreading for millimeter-sized droplets (e.g., Marsh et al. (1993), Eggers \& Stone (2004)). Adaptive mesh refinement (AMR) has been developed previously for flows with moving contact lines (Afkhami et al. (2009)) but appears not to have been utilized for the range of length scales required here. We have therefore integrated PARAMESH, a powerful adaptive mesh refinement software package for parallel computing (MacNeice et al. (2000)), into a level-set method for modelling axisymmetric flows with moving contact lines, resulting on a multi-processor-interfacebased (MPI) code. This method is presented in Sec. 2. Although we shall adopt a slip model formulation, the conclusion of Kafka \& Dussan V. (1979) that the meniscus shape is insensitive to the precise conditions near the contact line will also briefly be examined with the methodology developed here by comparing with results wherein a diffuse interface method is used without slip. Also, we do not assume the contact angle to be small.

In Sec. 3, we revisit the modelling of slow spreading. Although the shape of the interface in asymptotic analysis has been found to agree well with experiments (Marsh et al. (1993) for other slow contact-line flows), we first assess to what extent the numerical simulations agree with the well-established analysis of Hocking \& Rivers (1982) regarding the shape of the drop and the apparent contact angle; also, the analysis of slow spreading is partly used in that of rapid spreading (i.e., in the inner region). We also revisit the definition of the apparent angle as discussed above. Hocking \& Rivers (1982) used a matchedasymptotic expansion for slow droplet spreading wherein the solution for an inner region in the immediate vicinity of the contact line is matched to that in the outer region (the large-scale flow) by means of an intermediate expansion. The matching conditions then yield a relation between an apparent contact angle (that is used in the outer region) and a capillary number $C a \equiv \mu U / \sigma$ based on the instantaneous contact-line speed $U$, with the slip length made dimensionless with the drop diameter $\lambda$ explicitly as a parameter; to achieve this, the interface shape and velocity field are also determined in parts of the various regions. A detailed comparison of the interface shape with numerical simulation of the same problem is further desirable because of simplifying assumptions that have necessarily been made at some places in the analysis (this is discussed further in Sec. 3.1). We also include results for different viscosity ratios to supplement the analysis of Hocking \& Rivers (1982) wherein the outer fluid viscosity was ignored. Further aspects of the contact-line motion (including the structure of the flow field and the wall shear stress and pressure) are investigated after the results for the drop shape in rapid spreading have been presented.

A direct equivalent of the work of Hocking \& Rivers (1982) is not available for rapid droplet spreading/dewetting. The analysis of Cox (1986) for general slow contact-line motion has been extended to this regime by Cox (1998), however. The contact-line region is divided there into an outer, intermediate and inner region, where the intermediate region is divided further into a viscous and inviscid inner region (further details are briefly recalled in Sec. 4). An objective of Sec. 4 is to assess whether the various regions are indeed recovered (in particular, during the passage of a capillary wave) and if so, to determine from these the values of the various constants that were taken to be unity in the analysis of Cox (1998). In Sec. 5, we build upon these results and address various issues in droplet spreading: the structure of the flow field, the shear stress and pressure at the wall, and the extension of the analysis of Cox (1998) for dewetting. Finally, we consider the comparison with experimental data in Sec. 5.6. 


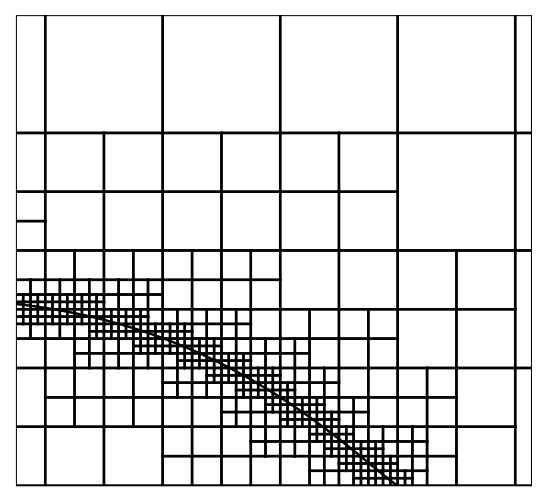

FiguRE 1. Illustration of the typical block structure in LS simulation of drop spreading with PARAMESH.

\section{Numerical method}

We consider here axisymmetric spreading and dewetting of a drop of diameter $D$ on a flat solid surface with homogeneous surface properties. The viscosity and density of the fluids are denoted by $\mu$ and $\rho$, and the ratios between the properties outside and inside the drop $r_{v}=\mu_{2} / \mu_{1}$ and $r_{d}=\rho_{2} / \rho_{1}$ will be used. Gravity is not considered in the present work (unless stated otherwise). The initial shape of the drop is chosen to be a circular cap with constant contact angle $\theta_{i n i}$, and a different microscopic contact angle $\theta_{w}$ is prescribed so that the drop will spread or dewet towards a shape corresponding to the microscopic contact angle. Based on the contact line speed, the instantaneous Reynolds number and capillary number are defined as $R e=\rho_{1} U D / \mu_{1}$ and $C a=\mu_{1} U / \sigma$ where $\sigma$ represents the interfacial tension between the two fluids.

A Navier slip boundary condition, $u_{r}=\lambda \partial u_{r} / \partial z$, is employed along the solid wall where $\lambda$ is the slip length. We assume that the problem is axisymmetric, and use a symmetry boundary condition at $r=0$. The component of the velocity gradient normal to boundaries are set as zero at the two remaining boundaries.

The level-set method used here is to some extent that of Spelt (2005), which is an extension of the method developed by Sussman et al. (1999) to account for moving contact lines. The differences with this earlier methodology are mainly that a finite volume method on a marker-and-cell (MAC) mesh is used, with velocity components defined at cell faces and scalar variables such as pressure and volume fraction at the cell centres. A fifth-order weighted essentially non-oscillatory (WENO) scheme (Liu et al. (1994)) is employed in the discretization of the advection term for the level-set function using the local flow velocity as the upwinding direction. More details regarding the numerical method and performance test can be found in Spelt (2005) and Ding et al. (2007b).

In order to simulate flows for more or less realistic values of a dimensionless slip length, $\lambda=O\left(10^{-4}\right)$, we have incorporated into our method the free open-source software package, PARAMESH (MacNeice et al. (2000)), which is an adaptive mesh refinement (AMR) tool developed for parallel computing. It is categorized into the structured and block-based adaptive mesh refinement family. The computational domain is covered with a hierarchy of grid blocks having the identical logical structure $(m \times n$ mesh). In the present study, the refining and coarsening of the grid blocks are based on whether the 
maximum distance of the grid block to the interface is smaller than a critical value. If true, a block is cut into two in all directions, respectively, producing four (in 3D this would be eight) children at a higher refinement level. Each of these has an identical logical structure, but the grid spacing in each direction is half that of its parent. Figure 1 shows an example of the typical block structure generated by PARAMESH for a drop spreading simulation. It can be seen that only the region which includes the interface and moving contact line is covered by fine mesh blocks. As it is also required that the jump in refinement level between two adjacent blocks is not larger than 1, the mesh density varies relatively smoothly apart from at the interface. All the blocks shown in Fig.1 are in fact covered with a $20 \times 20$ uniform mesh in the present study. Each block is surrounded by several layers of guard cells on each side, which protrude into the adjacent blocks. The connection between adjacent blocks and the implementation of boundary condition are realized by filling the guard with data from its neighbouring blocks or user-defined boundary conditions, depending on its physical position. In PARAMESH, all mesh blocks have the same logic structure at all levels of refinement. Hence, once the flow solver is developed for one grid block, it can be easily applied to all other blocks independent of the level of refinement. In addition, the different blocks can be distributed relatively equally to different CPUs in an MPI parallel environment, which can support large-scale simulations.

In order to check the accuracy and efficiency of the hybrid code, LS-PARAMESH, several test simulations have been carried out. One example is for axisymmetric spreading of a drop with an initial radius of 0.5 and contact angle of $\pi / 3$; a different microscopic contact angle is prescribed to be $\pi / 6$. Other parameters are chosen as $O h=0.1, \lambda=0.01$ and $r_{d}=r_{v}=0.1$. The computational domain is similar to that of Ding et al. (2007b), with a size of 1 in both directions; our tests shows doubling the domain size would lead to nearly identical results. Simulations have been carried out for both the LS method with a uniform mesh of $1281 \times 1281$ covering the whole computational domain, and with LSPARAMESH which employed five levels of mesh with the finest level equal to the uniform mesh resolution. This has resulted in a maximum difference in the instantaneous contactline radius of $0.4 \%$, which confirms that only the interfacial and contact line regions require such level of refinement. The global error in mass conservation for each phase is below $0.4 \%$ throughout both simulations. It must be noted that with adaptive mesh refinement, with typical mesh structure showed in Fig.1, the total number of grid points is only $6.8 \%$ of a corresponding uniform mesh. Results of a convergence study along with details of the discretization used in the subsequent sections are provided in the Appendix.

\section{Comparison with Hocking \& Rivers (1982) for slow spreading}

We first simulate the slow spreading regime and investigate the extent at which the asymptotic analysis of Hocking \& Rivers (1982) is confirmed, in terms of the interface shape and the apparent contact angle.

\subsection{Interface shape}

Here we investigate the interface shape in more detail for slow spreading (relatively large values of $O h$ ), in terms of the angle that the interface makes with the horizontal wall as a function of arc length to the contact line, $\theta(s)$. Numerical results for this angle are shown in Fig.2. These are compared with analytical predictions of Hocking \& Rivers (1982) for droplet spreading under creeping-flow conditions, which are summarized here first. 


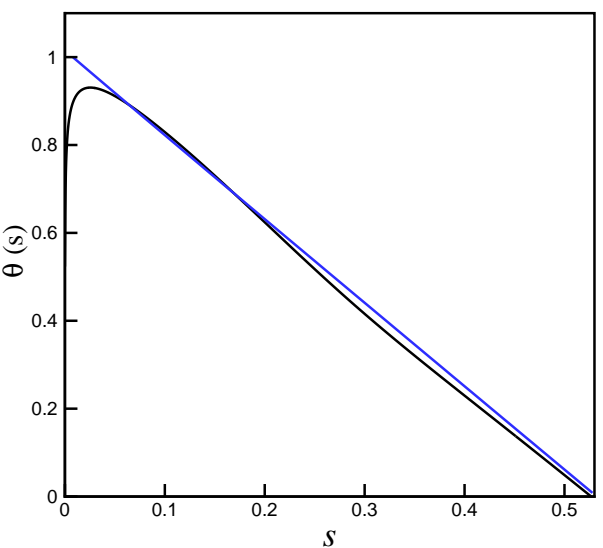

(a)

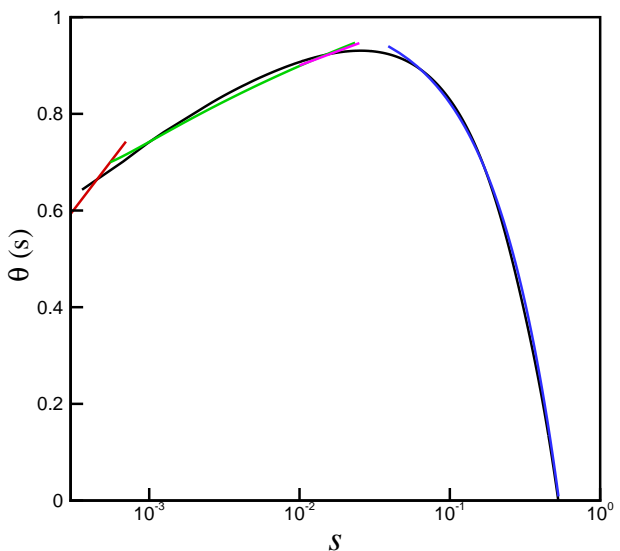

(b)

Figure 2. Droplet shape during spreading and definitions of macroscale angles: the angle that the interface makes with the wall as a function of arc length to the contact line, at the instance where $C a=0.016$ at $r_{d}=r_{v}=0.1, \lambda=0.0001, O h=0.1, \theta_{i n i}=\pi / 3, \theta_{w}=\pi / 6$. The colored lines represent the analytical result for the outer region far away from the contact line (3.1) (blue), the outer region near the intermediate region (3.4) (purple), the intermediate region (3.5) (green) and the inner region close to the intermediate region, (3.8) (red).

In the outer region, $\theta(s)$ follows from (e.g., Hocking \& Rivers (1982))

$$
\tan \theta(r)=\frac{a-r}{\left(a^{2} \operatorname{cosec}^{2} \theta_{a}-(a-r)^{2}\right)^{\frac{1}{2}}},
$$

where $a$ is the (time-dependent) radius of the contact line, and $r$ can be converted into $s$ through

$$
s=\int_{a}^{r} \frac{d r}{\cos \theta(r)}
$$

which is herein integrated numerically using the Maple software package. When comparing theory and simulations, the apparent angle $\theta_{a}$ in the theory (whose precise definition is discussed further in Sec. 3.2 below) is not taken from the simulations but instead from the theoretical prediction,

$$
g\left(\theta_{a}, r_{v}=0\right)=g\left(\theta_{w}, r_{v}=0\right)+C a\left(\ln \frac{a}{\lambda}-Q_{0}\left(\theta_{a}\right)+Q_{1}\left(\theta_{w}\right)\right),
$$

where the values of $Q_{0}$ and $Q_{1}$ have been tabulated in Hocking \& Rivers (1982) (their table 1). The only input in the theoretical prediction is therefore the value of the capillary number, $C a$, and the current radius of the contact line, $a$, alongside fluid properties.

Eq.3.1 simply corresponds to a hemispherical cap with the appropriate volume and apparent contact angle. Hocking \& Rivers (1982) have determined the correction to this in the outer region, in the limit wherein the intermediate region is approached. At this edge of the outer region

$$
\theta \sim \theta_{a}+\frac{2 C a \sin \theta_{a}}{\theta_{a}-\sin \theta_{a} \cos \theta_{a}}\left(\ln \left(\frac{s}{a}\right)+Q_{0}\left(\theta_{a}\right)+1\right) .
$$

which corresponds to Equations (3.20) and (5.9) in Hocking \& Rivers (1982). 
In the intermediate region, using inner region variables,

$$
g\left(\theta, r_{v}=0\right)=g\left(\theta_{w}, r_{v}=0\right)+C a\left(\ln \frac{s}{\lambda}+Q_{1}\left(\theta_{w}\right)+1\right),
$$

(corresponding to Equations (5.7),(5.10) upon using their (5.1), the relation $\epsilon=|1 / \ln \lambda|$ and $x=1+\epsilon \ln s=\epsilon \ln (s / \lambda)$ in Hocking \& Rivers (1982)) where

$$
g\left(\theta, r_{v}\right)=\int_{0}^{\theta} f\left(\beta, r_{v}\right) d \beta
$$

and

$$
f\left(\beta, r_{v}\right)=\frac{r_{v}\left(\beta^{2}-\sin ^{2} \beta\right)[(\pi-\beta)+\sin \beta \cos \beta]+\left[(\pi-\beta)^{2}-\sin ^{2} \beta\right](\beta-\sin \beta \cos \beta)}{2 \sin \beta\left[r_{v}^{2}\left(\beta^{2} \sin ^{2} \beta\right)+2 r_{v}\left[\beta(\pi-\beta)+\sin ^{2} \beta\right]+\left[(\pi-\beta)^{2}-\sin ^{2} \beta\right]\right]} .
$$

Note, this expression for $f\left(\beta, r_{v}\right)$ has been taken from Cox $(1986)$; it reduces to the corresponding expression by Hocking \& Rivers (1982), who restricted themselves to the case $r_{v}=0$, the generalised expression for arbitrary $r_{v}$ is stated here for future reference.

In the inner region, when approaching the intermediate region,

$$
\theta \sim \theta_{w}+\frac{C a}{g^{\prime}\left(\theta_{w}, r_{v}=0\right)}\left(\ln \frac{s}{\lambda}+Q_{1}\left(\theta_{w}\right)+1\right)
$$

(i.e., Equations (4.7) and (5.10) in Hocking \& Rivers (1982)).

Overall, reasonably close agreement is observed in Fig. 2 between the simulations and the analytical results, especially in light of the fact that the only input in the theory is (apart from fluid parameters such as the static angle and the slip length) the instantaneous value of $C a$ and $a$ (again, the apparent angle was not taken from the simulations). In Fig.2, the theory is presented for the value of $\mathrm{Ca}$ observed at the instance for which the numerical results are shown.

Some further observations can be made though. The inner and intermediate regions have been matched upon assuming that terms of $O\left(\theta_{a}-\theta_{w}\right)^{2}$ can be ignored, as is clear from comparing (3.5) with (3.8) (this can be seen from the fact that upon expanding $g(\theta)$ in $(3.5)$ about $\theta_{w}$, it only matches (3.8) when discarding terms of $\left.O\left(\theta_{a}-\theta_{w}\right)^{2}\right)$. The mismatch in slope between these two predictions seen in Fig.2 therefore hardly comes as a surprise.

A convenient definition of apparent angle corresponds to the value of $\theta(s=0)$ obtained from (3.1) (as, for instance, used by Hocking \& Rivers (1982) when comparing with experimental data; see also Fig.2(a)), although this is not an actual angle that can be taken from the full curve $\theta(s)$ in numerical simulations. Fortunately for the theoretical analysis though, the asymptotic expression (3.4) is found to match (3.5) very well for a large range of values of $\theta$, as long as a sufficiently large value is used - which is guaranteed by defining the apparent angle such that it exceeds all values along the interface. We also note here that for slow spreading the actual value of $\theta(s)$ at the edge of outer and intermediate region is in fact close to the maximum value of $\theta(s)$, which is the definition used in our previous work (Ding \& Spelt (2007a), Ding \& Spelt (2008), Ding et al. $(2010))$.

\subsection{Apparent contact angle}

In the work of Hocking \& Rivers (1982) an apparent angle is defined interchangeably as the angle at the edge of the outer region or as the angle that the interface makes with the wall in the outer region extrapolated to the contact line. As shown in the previous section, the difference between these values, although assumed small, is in practice not 
negligible, making the matching at the edge of the outer and intermediate regions somewhat peculiar. In any case, extrapolation of the outer-field shape to the contact line, and the local maximum value of the angle that the interface makes with the wall (which could be considered to be similar to the actual angle at the outer/intermediate boundary), may both be a convenient practical definition. In this section, we shall report results for the apparent angle defined in both ways.

Representative results from a parametric study for the apparent angle versus instantaneous capillary number are shown in Fig.3. The solid lines represent the results for the apparent angle defined as the maximum value of $\theta(s)$; dots those obtained by extrapolating the linear regime (seen in Fig.2a, for example) to $s=0$. Results are shown for different values of dimensionless slip length and viscosity and density ratio, $r_{v}$ and $r_{d}$, for $O h=0.1$, which would be expected to be sufficiently large for inertial effects to be small. We have found that the results for $r_{d}=r_{v}=0.05$, and for $r_{d}=1, r_{v}=0.1$ coincide almost exactly with those for $r_{d}=r_{v}=0.1$ in Fig.3d, these are therefore not shown for clarity of presentation. We conclude from this that within the range of parameters studied here, the density ratio has no effect (as would be expected in a creeping-flow limit), and that using $r_{v}=0.1$ (which is more efficient computationally) is sufficiently low to be useful as an approximation for the limit $r_{v} \rightarrow 0$.

The results in Fig.3 are also compared against the theoretical prediction (3.3) derived by Hocking \& Rivers (1982) for droplet spreading (note, this requires iteration). For $\lambda \leqslant$ 0.001 , the theory agrees very closely with the computational results for the extrapolated angle, even for capillary number values as large as 0.02 , whereas no adjustable parameters have been used. In prior work (e.g., Ding \& Spelt (2007a)), the maximum angle along the interface was used in comparisons against asymptotic theory; the results in Fig. 3 show that this will lead to an underprediction in the slow spreading regime. At relatively large slip length values, which is only relevant for very small drops, the asymptotic theory is not expected to agree well, as it requires sufficient separation of length scales, and the agreement with the simulation results when using the maximum angle along the interface at $\lambda=0.01$ is considered fortunate. Also, the theory predicts a much larger contact-line speed for a given extrapolated apparent angle (and vice versa). At the intermediate value $\lambda=0.001$ the simulations and theory already agree well for the apparent angle, but we have not found the detailed shape to be predicted as well as in Fig 2 for $\lambda=0.0001$.

An implication of this agreement is the following. For general contact line motion, Cox (1986) derived

$$
g\left(\theta_{a}, r_{v}\right)=g\left(\theta_{w}, r_{v}\right)+C a \ln \left(\frac{1}{\epsilon_{v}}\right) .
$$

This represents (7.20) in Cox (1986); the various terms on the r.h.s. there - including $Q_{0}^{*}, Q_{i}^{*}$ etc.- have been combined here in the present logarithmic term, i.e., our $\epsilon_{v}$ is proportional to $\epsilon$ (the ratio of slip length and a macroscale) used by Cox (1986). It was noticed by Cox (1986) that the precise value of $\epsilon_{v}$ (i.e., related to his function $Q_{0}^{*}$ etc.) could be dependent on the specific flow considered. Now, (3.3) can be put in the form (3.9), so we conclude that the proportionality constant in the relation between $\epsilon_{v}$ and the ratio of slip length and macroscale length scale should be chosen in accord with the analysis of Hocking \& Rivers (1982) in order to obtain good agreement with the computations. We have found the theoretical prediction (3.9) to be sensitive to the precise value of $\epsilon_{v}$; for instance, using the argument in the log term based on the lubricationtheory result of Hocking (1983) yields inferior results here. In many previous studies, the approximated form (corrected to $\mathrm{Ca}^{0}$ ) of Cox (1986) has been used with $\epsilon_{v}=\lambda$. Tests show this approximation overpredicts the apparent angle: typically we find $\epsilon_{v}$ to 


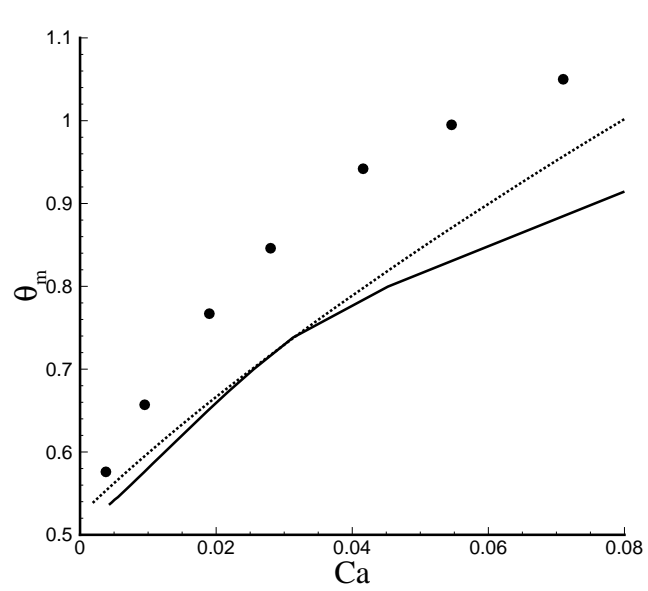

(a)

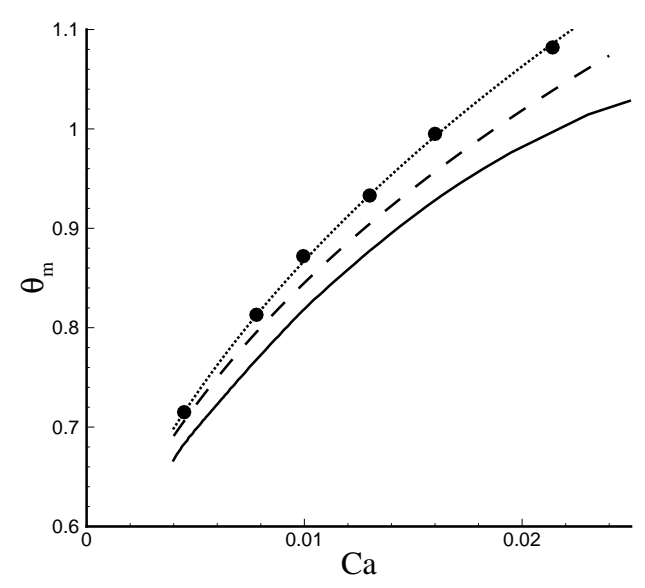

(c)

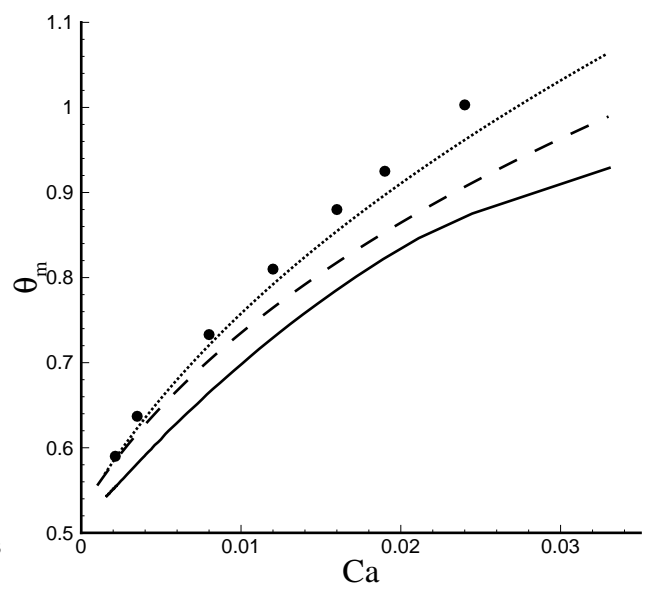

(b)

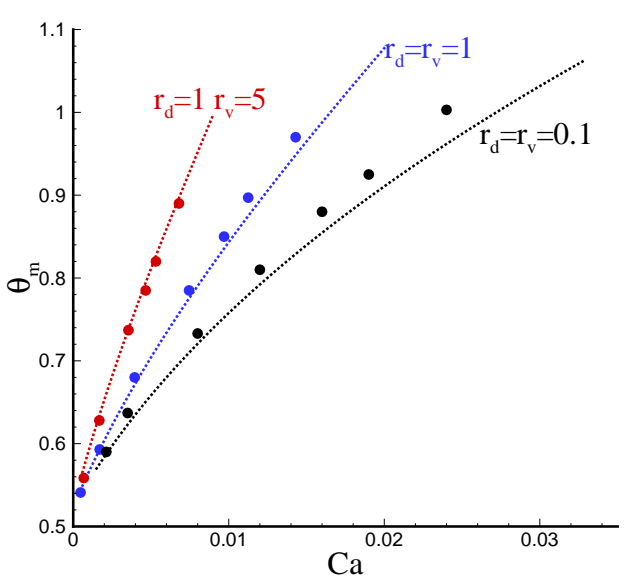

(d)

FIGURE 3. Parametric study of apparent contact angle as a function of instantaneous $\mathrm{Ca}$. Solid lines are simulation results, dotted lines are Eq.(3.3), dashed lines represent the lubrication theory of Hocking (1983); the dots in (a) - (c) represent results from the same numerical simulation but using a different definition of apparent angle, as explained in the text. Parameter values used are: $r_{d}=r_{v}=0.1, O h=0.1, \theta_{i n i}=\pi / 3, \theta_{w}=\pi / 6, \lambda=(a) 0.01 ;(b) 0.001 ;(c) 0.0001$. In (d), the effect of viscosity is shown for $\lambda=0.001$ (the other parameters are indicated in the figure).

be $O(10)$ times the slip length divided by the drop diameter. This should therefore be accounted for in order-of-magnitude estimates of an effective slip length if these are based on a relation such as $(3.9)$.

The results are also used in Fig.3 to determine the accuracy of the lubrication approximation, which is widely used in studies of slow spreading problems. The lubrication theory of Hocking (1983) is seen in Fig.3 not to deviate far from the simulations and the theory of Hocking \& Rivers (1982) (wherein lubrication theory is not used), especially at low capillary numbers.

Cox (1986) accounted for a finite viscosity ratio $r_{v}$ of the two fluids in his derivation of 
(3.9). Given the sensitivity of the theoretical results to the precise value of $\epsilon_{v}$, a possible concern may be that $\epsilon_{v}$ is affected by $r_{v}$. The study of Hocking \& Rivers (1982) does not provide an answer here, as the surrounding fluid is not resolved. But in Fig. 3(d) we see that the effect of viscosity ratio of the two fluids on the apparent angle is very well represented by (3.3) in conjunction with (3.7). Therefore, the viscosity ratio is not found to much affect the value of $\epsilon_{v}$ in (3.9), but to substantially change $g\left(\theta_{a}, r_{v}\right)$.

Simulations with the initial angle $\theta_{i n i}$ increased significantly, up to $2 \pi / 3$, have been carried out with other parameters similar to those in Fig. 3(b). The result in terms of the apparent angle as a function of instantaneous capillary number (not shown) agrees very well with the asymptotic result of Hocking \& Rivers (1982).

\section{Validation and refinement of theory of Cox (1998) for fast spreading}

So far, the results have been for slow spreading. In this section, we consider smaller values of $O h$, corresponding to larger inertial effects (we recall that $R e=C a / O h^{2}$, so $R e$ is then larger at a fixed $\mathrm{Ca}$ ). We shall compare results for the interface shape and apparent contact angle with a modified form of the analysis of Cox (1998) for spreading in the regime $1 \ll R e \ll \lambda^{-1}, C a \ll 1$, which we briefly introduce and review first.

\subsection{Cox (1998) revisited}

In this regime, Cox (1998) divided the contact-line region into inner and outer regions, as in the viscous analysis (Cox (1986)), and two intermediate subregions, viscous and inviscid, that match in a transitional zone. The transition is at a distance from the contact line $d^{*}$ chosen such that a Reynolds number based on the contact-line speed and $d^{*}$ is of $\mathrm{O}(1)$. Cox (1998) took this Reynolds number to be exactly unity, i.e., $d^{*}=1 / R e$, but here, noting the liberty in the choice of length scale in the definition of a Reynolds number (drop radius, diameter, or otherwise), as well as following initial comparisons against numerical simulations, we propose instead to adhere to a transition Reynolds number $R e^{*} \equiv d^{*} R e$ that is of $\mathrm{O}(1)$. In the viscous intermediate sublayer, the angle $\theta$ between the interface and the wall as a function of distance $d$ to the contact line is given by

$$
g(\theta)-g\left(\theta_{w}\right)=C a \ln (d / \epsilon)+C a Q_{i} f\left(\theta_{w}\right), \quad \epsilon<d<R e^{*} / R e .
$$

which is essentially the same as the viscous analysis of Cox (1986). At the edge $d^{*}=$ $R e^{*} / R e$, the interfacial angle $\theta^{*}$ follows from

$$
g\left(\theta^{*}\right)-g\left(\theta_{w}\right)=C a \ln \left(R e^{*} /(\operatorname{Re} \epsilon)\right)+C a Q_{i} f\left(\theta_{w}\right),
$$

In the limit of $r_{v} \rightarrow 0, Q_{i} f=1+Q_{1}$ where $Q_{1}$ can be found in table 1 of Hocking \& Rivers (1982). This term $Q_{i}$ was included at an early stage in the analysis of Cox (1998) (denoted by him as $Q_{v}^{*}$, and as $Q_{i}^{*}$ in Cox (1986)), in his Eq. (8.19), but he eventually dropped all terms of order $\mathrm{Ca}^{+1}$ for simplicity. Here we have reinstated this term as we have found this to have an effect on the results that cannot be ignored. The parameter $\epsilon$ in Eq. 4.2 is of $O(\lambda)$. In fact, as the inner region and viscous intermediate sublayers in the inertial theory are the same as in slow spreading, it is expected that $\epsilon=\lambda$, as was used in the model for slow spreading which is shown in the preceding section to agree well with the numerics (strictly speaking, a small deviation from this is allowed, as the matching condition is only to be applied close to the boundary between the inner region and intermediate viscous sublayer). This is confirmed below also for fast spreading. 
In the inviscid subregion, the interface shape is given by

$$
g_{i v}(\theta)-g_{i v}\left(\theta^{*}\right)-C a\left[h_{i v}(\theta)-h_{i v}\left(\theta^{*}\right)\right]=C a \ln \left(d R e / R e^{*}\right), \quad d^{*}<d<1
$$

where

$$
\begin{gathered}
g_{i v}(\theta)=1.53161(\theta-\sin \theta) \\
h_{i v}(\theta)=-2 \ln \left(\sin \left(\frac{1}{2} \theta\right)\right)+2 \int_{\pi}^{\theta} \frac{\theta d \theta}{1-\cos \theta}
\end{gathered}
$$

As in the viscous subregion, Cox (1998) did determine the term $O(\mathrm{Ca})$ included in the above, but later left out all terms of this order for simplicity; we have re-instated these, also for consistency with the term of the same order in (4.2) above. The theoretical prediction is compared below against the numerical simulations.

An apparent angle $\theta_{m}$ follows from matching the inviscid subregion to the outer region. In the outer region, Cox (1998) defined the apparent angle such that it is attained at a distance of one unit length from the contact line (cf. his Eq.3.39). Matching then results in the requirement that (4.3) returns $\theta_{m}$ as $d \rightarrow 1$, yielding

$$
g_{i v}\left(\theta_{m}\right)-g_{i v}\left(\theta^{*}\right)-C a\left[h_{i v}\left(\theta_{m}\right)-h_{i v}\left(\theta^{*}\right)\right]=C a \ln \left(R e / R e^{*}\right) .
$$

However, the limit $d \rightarrow 1$ is not physically useful here, since the macroscopic length scale used in normalizing variables was not unambiguously defined by Cox (1998). Furthermore, it is not expected to yield an angle that can be observed anywhere on an interface, and is found here not to be an accurate approximation of the angle that would be obtained by measuring instead an extrapolation of the interfacial angle from the outer region to the contact line. Therefore, we insist herein on defining $\theta_{m}$ as the interfacial angle extrapolated from the outer region. This apparent angle is attained at some value of $d=d_{m}=O(1)$, hence the insistence on a meaningful definition of $\theta_{m}$ comes at the cost of having to replace (4.6) with

$$
g_{i v}\left(\theta_{m}\right)-g_{i v}\left(\theta^{*}\right)-C a\left[h_{i v}\left(\theta_{m}\right)-h_{i v}\left(\theta^{*}\right)\right]=C a \ln \left(d_{m} R e / R e^{*}\right) .
$$

A comparison between theory and numerics is used below to find $d_{m}$.

\subsection{Interface shape}

In Fig.4, results are shown for a reduced value of $O h=0.01$. A capillary-type wave is known to travel from the contact line towards the top of the droplet and then returns to the contact line (e.g., Ding \& Spelt (2007a), Ding et al. (2012)), but it has hitherto not been possible to assess how and to what extent this wave intrudes into and bounces back from the asymptotic structure of the contact-line region. In fact, a direct comparison with Cox (1998) appears unavailable so far even if capillary waves are far away from the contact line.

We address this latter issue first, as presented in Fig.4c. The interface profile in the intermediate viscous sublayer can be obtained from (4.1). The analytical results are in terms of the distance $d$ to the contact line rather than the arc length, $s$, but we have found this to have no visible effect on the results shown. We find that taking $\epsilon=1.5 \lambda$ (blue curves) leads to excellent agreement between the theory and simulation for all cases tested, and that $\epsilon=\lambda$ only generates a deviation of less than one degree. We thus conclude that in Cox (1998), taking the value of $\epsilon$ close to $\lambda$ is a reasonable choice. The shape of the interface predicted in the intermediate inviscid sublayer (green line) is only meaningful when the capillary wave is far away from the contact line, however. A proper value of $R e^{*}$, which is always of $O(1)$, is needed for the theory to agree closely with the simulations. We have generally found $R e^{*}$ to range from 0.2 to 1 here. 


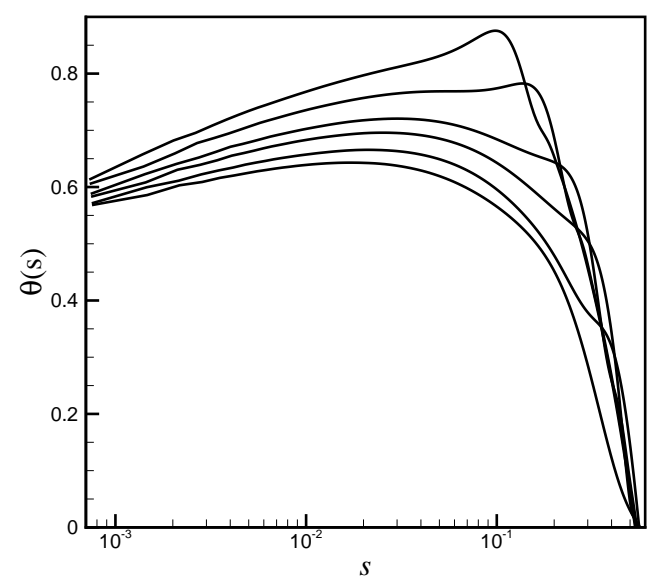

(a)

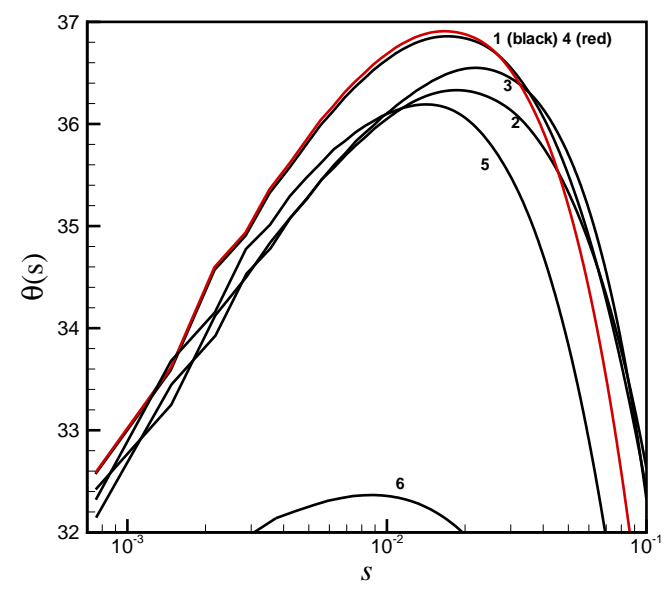

(b)

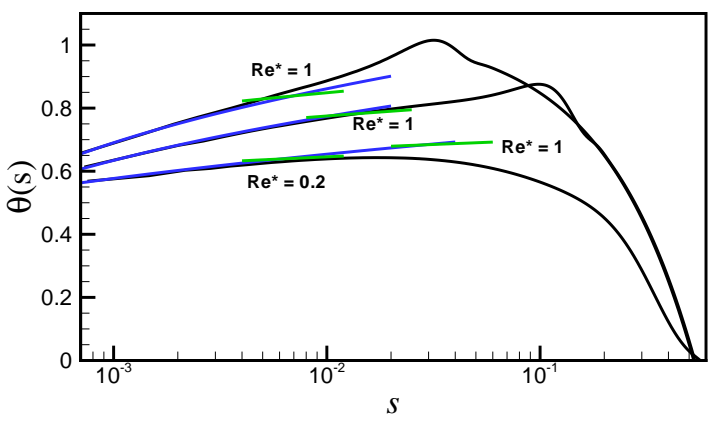

(c)

Figure 4. Angle that the interface makes with the wall as a function of the arc length to the contact line. (a) Capillary waves travel towards the top of the drop. From top to bottom, $(t, C a)=(0.006,0.01),(0.011,0.0083),(0.021,0.0068),(0.031,0.0059),(0.051,0.0049)$, $(0.071,0.0042)$. (b) Capillary-type waves bounces back and reach the contact-line region. Successive labels from 1 to 6 refer to $(t, C a)=(0.071,0.0042),(0.076,0.0038),(0.086,0.0037)$, $(0.096,0.0042),(0.106,0.004),(0.136,0.0016)$. (c) Comparison between simulation and Cox (1998). Blue curves are for intermediate viscous sublayer from (4.1) and (4.2); green lines are for intermediate inviscid layer from (4.3). Flow parameters are: $O h=0.01, r_{d}=r_{v}=0.1$, $\lambda=0.0001, \theta_{\text {ini }}=\pi / 3, \theta_{w}=\pi / 6$.

The time at which the capillary-type wave reflects back and reaches the contact line is captured in Fig.4b. Inspection of the sequence of snapshots shows two main trends: the oscillatory motion of the contact line, and of the outer region, which are nearly in phase. Indeed, a simple model can be constructed to approximate these profiles, by using (4.1) with the actual (oscillating) contact-line speed, and subtracting a term (such as a simple power of $s$ with an oscillating coefficient of proportionality) to represent the outer region, and taking the two amplitudes to oscillate in phase.

Results at an even lower value of $O h=0.00316$ are shown in Fig.5. Evidently, the capillary-type wave is now more prominent. Especially at very early times, when the capillary wave leaves the contact line, propagating through the contact-line region, the 


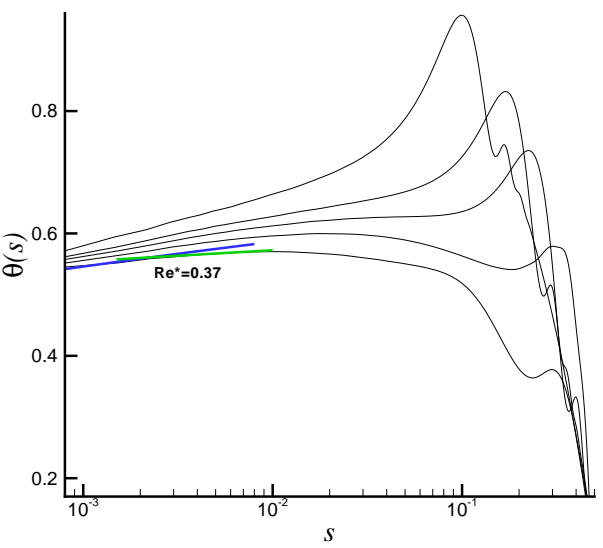

(a)

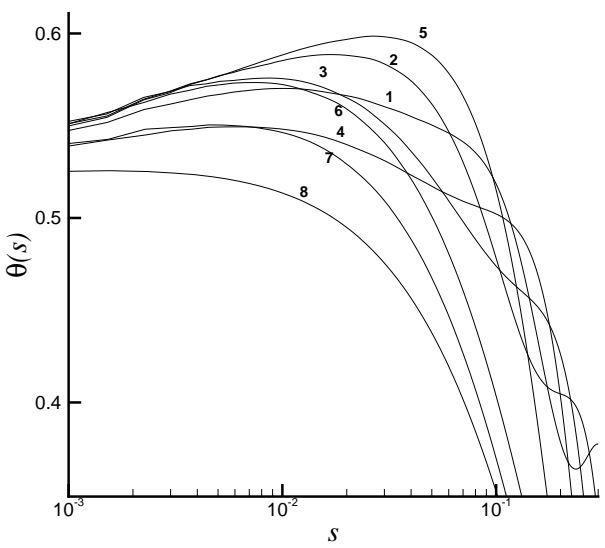

(b)

Figure 5. Droplet shape at low $O h(O h=0.00316)$. Shown is the angle that the interface makes with the wall as function of the arc length to the contact line, $\theta(s)$ at early times (a) and at the stage where a capillary wave has reached the contact-line region (b). In (a), results are shown for successive times (from top to bottom) at times when $C a=0.00441,0.00338,0.00295,0.00247$, 0.00186 . Blue curves are for intermediate viscous sublayer from (4.1) and (4.2); green lines are for intermediate inviscid layer from (4.3). In (b), successive labels refer to $C a=0.00186,0.00195$, $0.00215,0.00124,0.00184,0.00218,0.00136,0.00029$. Other fluid parameters are: $r_{d}=r_{v}=0.1$, $\lambda=0.0001, \theta_{\text {ini }}=\pi / 3, \theta_{w}=\pi / 6$.

$\theta(s)$ curve is pulled up in the inviscid sublayer more than is predicted by the theory. Again though, the theory of Cox (1998) for the intermediate viscous and inertial sublayers works very well when the capillary wave is not close to the contact line. The behaviour around the time when the capillary wave has again reached the contact line appears more complex than at $\mathrm{Oh}=0.01$ (cf. compare Fig.5b with Fig.4b). Not one but two periods of oscillation can now be observed (close inspection of time signals has shown a third), but the outer region and the contact-line speed remain nearly in phase, as they are at $O h=0.01$ above (we return to this in the next subsection).

\subsection{Apparent contact angle}

We now investigate the apparent contact angle as a function of the contact-line speed, which is shown in Fig.6a-b for $O h=0.01$ and in Fig.6c-d for $O h=0.00316$. Results for two definitions of the apparent angle are shown in each case; the solid lines represent the apparent angle defined as the maximum value of $\theta(s)$, and dots represent $\theta(s=0)$ obtained by extrapolation. With either definition, loops are observed, showing non-uniqueness in the apparent angle versus the instantaneous contact-line speed. This behaviour is consistent with that observed in Ding \& Spelt (2007a) (see also Sec. 5.6); we note that this figure illustrates also the behaviour discussed in the previous subsection, as it is clear that the apparent angle and contact-line speed oscillate in phase. Here however, our interest lies in comparing with theory, which was not possible in that earlier work. In Fig. 6, the results labelled Cox (1986)A correspond to Cox (1986) corrected to $\mathrm{Ca}^{0}$ $\left(\epsilon_{v}=\lambda\right.$ in (3.9)); Cox (1986)B represents Cox (1986) corrected to $\mathrm{Ca}^{+1}\left(\epsilon_{v}\right.$ chosen in accord with the analysis of Hocking \& Rivers (1982) which accounts the terms including $Q_{0}{ }^{*}, Q_{1}{ }^{*}$ etc.). It is seen that both overpredict the apparent angle. With Cox (1998) corrected to $C a^{0}\left(Q_{i}=0\right)$, and setting $R e^{*}=1$ and $d_{m}=1$ as done in Cox (1998), the analysis (the curve labelled $\operatorname{Cox}(1998) \mathrm{A}$ ) also leads to an overprediction. It is argued here that this is caused by the fact that the parameters such as $R e^{*}$ in the theory are 


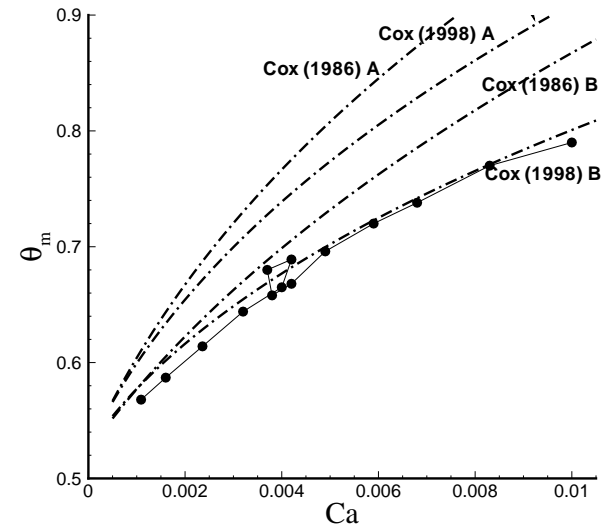

(a)

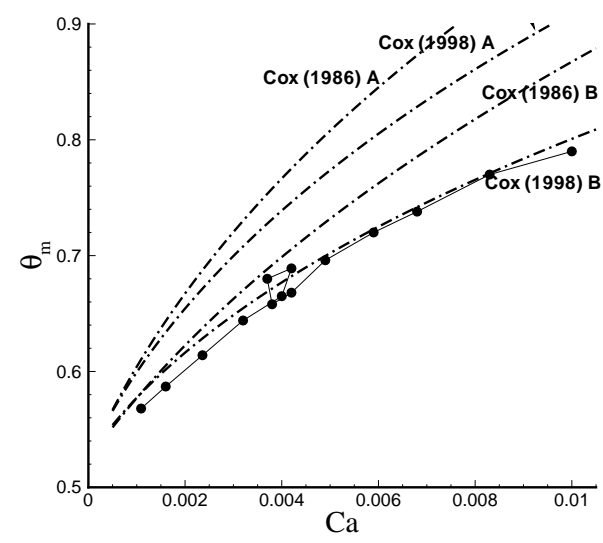

(c)

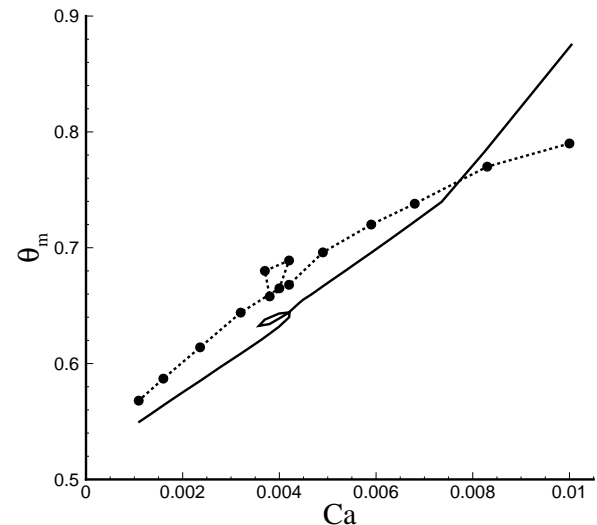

(b)

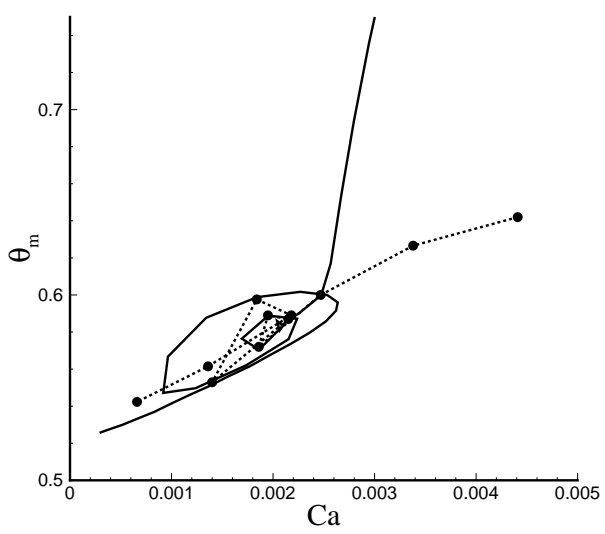

(d)

Figure 6. Apparent contact angle as a function of instantaneous $\mathrm{Ca}$ from simulation and theory. $\lambda=0.0001, O h=(\mathrm{a}, \mathrm{b}) 0.01,(\mathrm{c}, \mathrm{d})$ 0.00316. The symbols represent the simulation results when using the extrapolated angle; in (b) and (d), the solid lines represent the simulation results when using the maximum angle along the interface. The dash-dotted lines in (a) and (c) represent the analytical results, as explained in the text.

of $O(1)$ but not exactly unity, as discussed in the previous subsection on the interface shape. We find reasonably good agreement when using Cox (1998) corrected to $\mathrm{Ca}^{+1}$ (the data labelled Cox (1998)B in Fig.6), where the value of $R e^{*}$ is obtained by taking an average over values obtained by fitting various instantaneous interface shapes, which gives a value of about 0.37 here). We also find $d_{m}=0.2$ should be used in order to achieve good agreement for all cases considered herein.

Droplet spreading with even stronger inertial effects have been investigated recently by Ding et al. (2012), identifying a regime wherein small droplets pinch off due to rapid spreading motion, both numerically, using a diffuse interface method, and experimentally. Experimental and numerical results for the apparent angle vs. the dimensionless contactline speed were included, with the apparent angle used defined as the maximum angle following earlier work (Ding \& Spelt (2007a)). It was found that the numerical results were of the same order of magnitude, but could not be represented by the theory of 


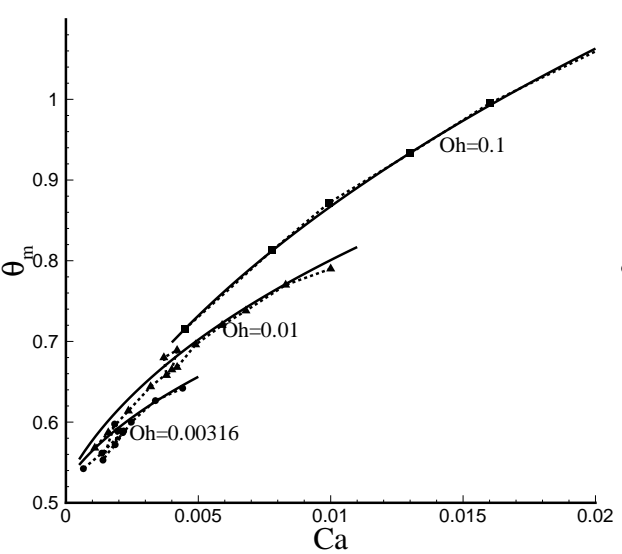

(a)

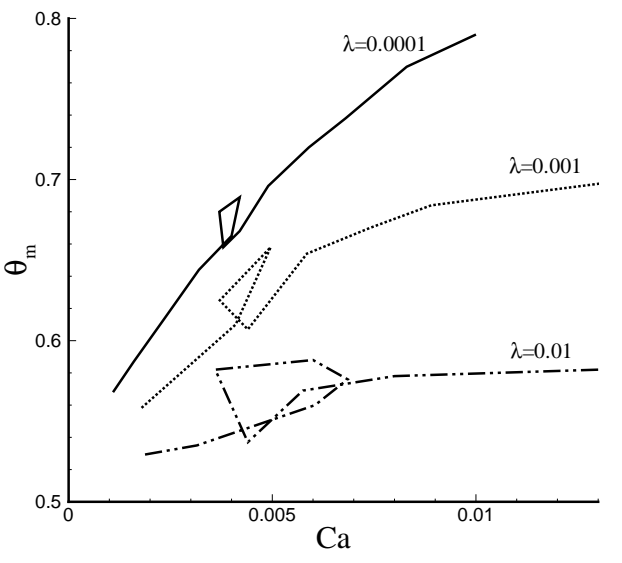

(b)

FIGURE 7. Apparent contact angle defined by extrapolation as a function of instantaneous $C a$ for (a) $\lambda=0.0001$ with different $O h$ (zoomed views for low $O h$ are in Fig. 6) and (b) $O h=0.01$ with different $\lambda$.The solid lines in (a) represent Cox (1998)B except for $O h=0.1$ which is compared with Hocking \& Rivers (1982).

Cox (1986) or Cox (1998). However, we have now verified that when obtaining in those simulations the apparent angle by extrapolation from the outer region to the contact line, good agreement with Cox (1998) (4.2 and 4.7) can be achieved, with the values of $R e^{*}$ and $d_{m}$ chosen in the same way. From Fig.6b and d, we conclude that for fast droplet spreading, using the maximum interfacial angle to approximate the apparent angle leads to a significant overestimate.

We summarize the extrapolated apparent angle as a function of contact line speed for both slow and fast spreading in Fig.7a. It is clear that inertial effects tend to decrease the apparent angle, as predicted by Cox (1998). This reduction of apparent angle due to inertial effects also agrees with the experimental finding of Stoev et al. (1999).

Finally, we revisit the problem of identifying the effect of slip length on drop spreading when inertial effects are important. As summarized in the introduction, the prior work on simulation of droplet spreading is strongly restricted by the rather large value of the equivalent slip length. The slip length studied herein ranges from $10^{-2}$ to $10^{-4}$; Fig.7b presents the apparent contact angle as a function of instantaneous capillary number. It is seen that at the same apparent contact angle, the contact line moves faster with a larger slip length. If the slip length is large, the contact line spreading rate shows dampened oscillations in the initial transient stage and capillary waves travel along the interface, and the apparent angle is not a single-valued function of $C a$, as alluded to previously. Upon decreasing the slip length, the contact line moves more slowly so that this oscillatory behaviour is less significant. It is seen in Fig.7b that the loop in the curve become smaller upon decreasing the slip length. Although it is expected that the drop will spread monotonically for a sufficiently small value of the slip length at a fixed value of $O h$, this can be compensated by reducing the value of $O h$, as can be seen in Fig.7a.

\section{Further discussion of results}

In this section we make further use of the simulations presented above by revisiting the discussion of several issues in droplet spreading. 


\subsection{Flow field}

The experiments of Dussan V. \& Davis (1974) have shown the fluid to be rolling as a contact line advances, with an 'ejection' or 'injection' separation streamline in the other fluid when using the frame of reference moving with the contact line; Chen et al. (1997) have confirmed the rolling-type motion down to distances of $O(\mu \mathbf{m})$ from the contact line. Analysis of the contact-line region for slow contact-line motion in the absence of slip results in a wedge-flow pattern with, depending on the viscosity ratio and static contact angle, the ejection/injection separation streamline predicted to be in the less viscous fluid unless the contact angle is very large (e.g., Huh \& Scriven (1971)). A similar result was obtained in the intermediate layer in the slow regime with a contact-line model such as slip (Cox (1986)). Although the slip model results in a flow akin to stagnation-point flow around the contact line, without a finite arrival time at the contact line of fluid particles along the interface, whereas pure rolling does result in such a finite arrival time, the confirmation by experiment of the velocity field in the inner region poses a formidable challenge. But the experiments of Savelski et al. (1995) and Fuentes \& Cerro (2005) show discrepancies with this hydrodynamic theory regarding the flow field even outside what would be expected to be the inner region. Also, in the numerical simulation of Sheng \& Zhou (1992), a vortex is observed in the wedge near a contact line when applying free slip in the immediate vicinity of the contact line and no slip elsewhere.

To investigate whether the present computations are consistent with the asymptotic analysis of slip models, and to see whether for instance inertial effects could play a role here, we have analyzed the flow field near the moving contact line during drop spreading in our simulations. All of our simulations of droplet spreading show a vortical pattern in the surrounding fluid near the contact line; a typical example is shown in Fig.8a, which is discussed further below. A zoomed view of the same field, showing the contactline region is presented in Fig. 8b. This is seen to correspond to the typical wedge flow pattern also predicted by analysis (e.g., Huh \& Scriven (1971)); we have also verified that the velocity along the interface uniformly goes to zero when the contact line is approached in a further high-resolution simulation (the case with finest mesh in Fig. 17), within the accuracy of the determination of the contact-line speed. The splitting-ejection streamline is found on the side of the less-viscous fluid. We have determined the angle that this streamline makes with the wall for various values of the viscosity ratio and the Ohnesorge number, the results are shown in Fig. 8c. We find that this angle remains virtually unchanged during the spreading. The results for cases wherein spreading is sufficiently gradual $(O h=0.0316$ and 0.1$)$ agree with the theoretical prediction of Huh \& Scriven (1971). For $r_{v}=5$, both the theory and the present simulation predict a separation streamline inside the 'drop'.

We also find that inertia affects the angle that the separation streamline and the wall make: in Fig. 8c, the angle is somewhat smaller if $O h$ is decreased by a further order of magnitude. Such reduction in the angle between the wall and the ejection streamline is found to result in a corresponding change in the extent of the vortical region seen in Fig.8a: its dimension perpendicular to the interface is found to be reduced when inertial effects become important.

\subsection{Shear stress and pressure along the wall}

The total force exerted by the flow on the wall is of practical interest (e.g., in the conditions for the depinning of droplets by shear, Spelt (2006), Ding \& Spelt (2008), Ding et al. (2010)). In Fig. 9 the shear stress and pressure on the substrate are presented as a function of distance to the contact line in a case of slow spreading.

In the inner region, the shear rate at the wall is $U_{c l}(a-r)^{-1} k_{1}(r)$ where $k_{1}(r)$ can be 

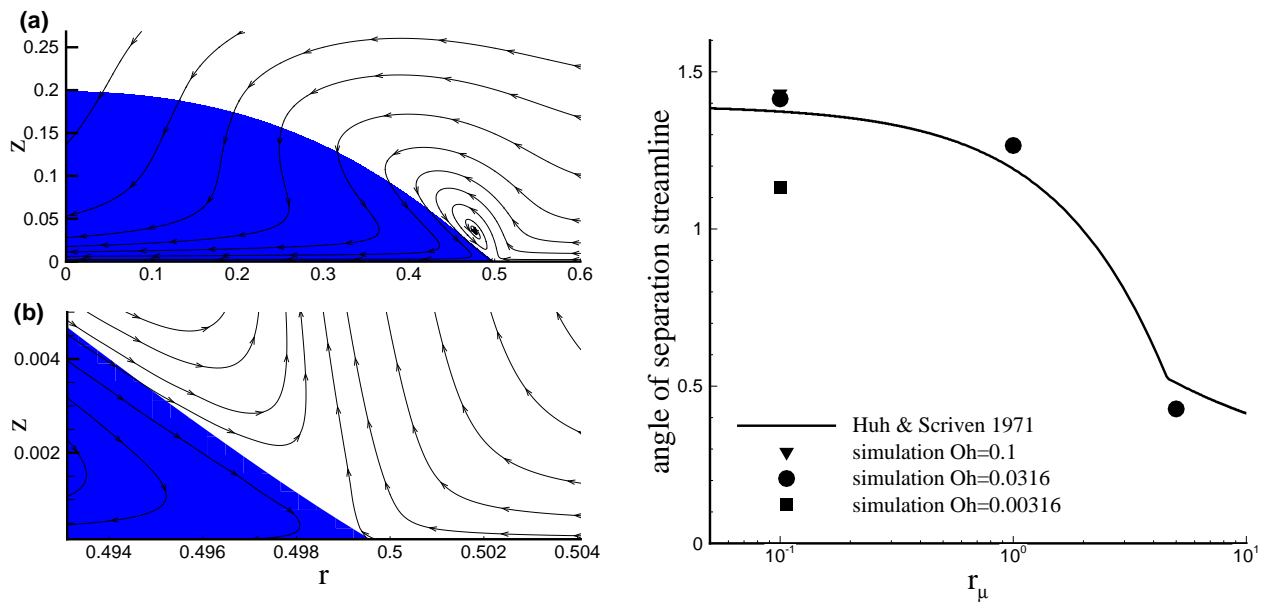

(c)

FIGURE 8. (a) Streamline pattern around the contact line during spreading (in a frame of reference moving with the contact line). $\lambda=0.001$, $O h=0.0316, r_{d}=r_{v}=0.1, \theta_{\text {ini }}=\pi / 3, \theta_{w}=\pi / 6$. (b), A zoomed view of (a) near the contact line. (c) Angle of separation streamline as a function of viscosity ratio, including results for different values of $\mathrm{Oh}$ as indicated in the figure.

obtained by solving an integral equation numerically (Hocking (1977), Hocking \& Rivers (1982); we note here that $k_{1}$ diverges as $\lambda \rightarrow 0$ ). In the intermediate region, the result is of the same form but with $k_{1}(r)$ set equal to $\partial^{2} g_{H R} / \partial \theta^{2}$ where $g_{H R}$ is the function $g$ in Hocking \& Rivers (1982). In the outer region, we have obtained the wall stress from the stream function (which is given in bipolar coordinates $(\xi, \eta)$ ) as $\partial^{2} \psi / \partial \xi^{2}$ at $\xi=0$, which requires a numerical integration that was conducted in the software package Maple, as it involves further integration of a Legendre polynomial of partial complex order. In Fig. 9(a), the present simulation results are seen to agree very well with the analysis in all regions.

The pressure along the wall is not readily obtained from such asymptotic analysis. The exception is the pressure in the intermediate region. We have obtained this by determining the vorticity $\zeta$ from the stream function through $\nabla^{2} \psi=\zeta$, and hence the pressure along the wall by numerically integrating $\partial p / \partial r_{1}=-r_{1}^{-1} \partial \zeta / \partial \theta$ at $\theta=0$, where $r_{1} \equiv(a-r) / \lambda$ when $\theta=0$. The present results agree well with this, see Fig. 9(b).

A deviation from the result for the pressure in the intermediate region is of course observed when the inner region is approached. An explicit result for the pressure in the inner region is not derived in Hocking \& Rivers (1982), although it is clear from the fact that in Stokes flow, the pressure satisfies Laplace's equation, $\nabla^{2} p=0$, and $p \sim U_{c l} \ln (a-r)$ (in dimensionless form). It appears not possible to obtain the proportionality constant directly from the analysis of Hocking \& Rivers (1982). An approach to such behaviour is consistent with the results in Fig. 9(b). This is clarified further in the convergence study reported in the Appendix. It is demonstrated there that the inner region must be resolved such that the grid spacing is much smaller than the slip length before this logarithmic regime is clearly observed, even though the shape of the interface has converged already when the grid spacing is of the same order as the slip length. We infer from Figs. 16,17 the proportionality constant mentioned above to give for $\theta_{w}=\pi / 6, p \approx c U_{c l} \ln (a-r) / \lambda$ 


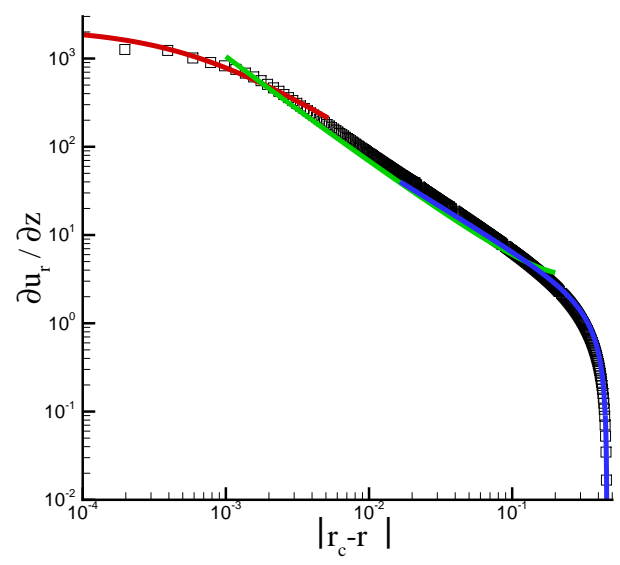

(a)

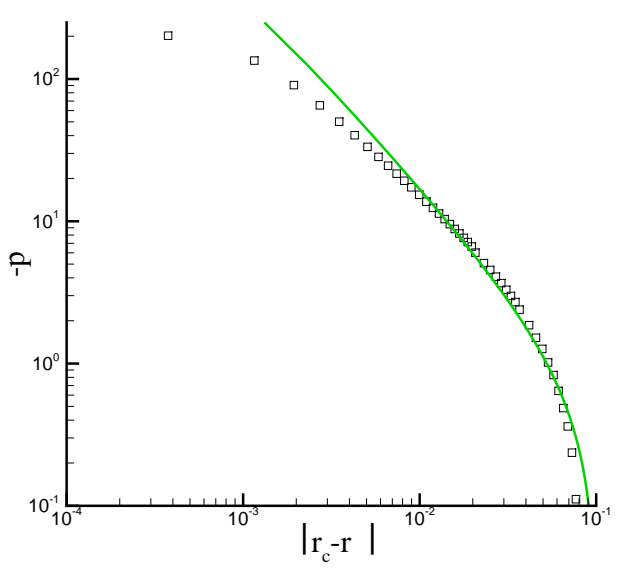

(b)

FIGURE 9. Comparison of instantaneous (a) shear rate and (b) pressure along the wall with Hocking \& Rivers (1982) for slow spreading, with $r_{c}$ denoting the contact-line radius; $r_{d}=r_{v}=0.1$, $\lambda=0.0001, O h=0.1, C a=0.011, \theta_{i n i}=\pi / 3, \theta_{w}=\pi / 6$. The three line segments in (a) represent (from left to right) the inner, intermediate and outer region. In (b) the line represents a result inferred from Hocking \& Rivers (1982) for the intermediate region (a complete result for the pressure is not given in Hocking \& Rivers (1982) in the inner and outer regions).

with $c=0.1 \pm 0.05$, or, in dimensional form,

$$
p_{\text {wall }}=c \hat{\mu} \hat{\lambda}^{-1} \hat{U}_{c l} \ln ((a-r) / D),
$$

where the hat decoration indicates dimensional values. A parametric study to determine the dependency of the prefactor $c$ on the contact angle has not been undertaken here, but it is noted that from lubrication theory this is expected to be proportional to $\theta_{w}^{-1}$ (e.g., Devauchelle et al. (2007)).

It is demonstrated in Sec. 4 above that the shape of the interface in the inner region for rapid spreading is virtually the same as in slow spreading. Inertial effects are not expected on this small scale, and we have found no evidence to the contrary (in fact, the convergence study in the Appendix is for modest inertial effects, $O h=0.0316$ ). It is conceded that in an extreme superspreading regime, inertial effects could even be important on the slip length scale (this regime is also studied by Cox (1998)), but this requires a very small contact angle not simulated herein.

\subsection{Sensitivity to contact line model}

Ding \& Spelt (2007a) have shown that the results obtained with a slip-length-based level-set method can agree well with those of a diffuse interface method, which relies on limited diffusion between the fluids concerned to alleviate the stress singularity at a moving contact line and utilizes a no-slip condition. Reasonable agreement was found when making a suitable choice for the diffuse interfacial thickness, measured by $\zeta$, for a given slip length. It was found that when $\zeta / \sqrt{\mu M}=O(1)$, where $M>0$ is the mobility which can be considered as a diffusion coefficient of chemical potential, $\lambda=\eta \zeta$; for $\lambda=O(0.01)$, Ding \& Spelt (2007a) found $\eta=1.8 \pm 0.2$ to give the best comparison. This has now been extended to much smaller values of $\lambda$ and $\zeta$. A direct comparison is shown in Fig.10, exhibiting good agreement in droplet shape and in the apparent angle as a function of contact-line speed. These new results indicate that the coefficient $\eta$ would 


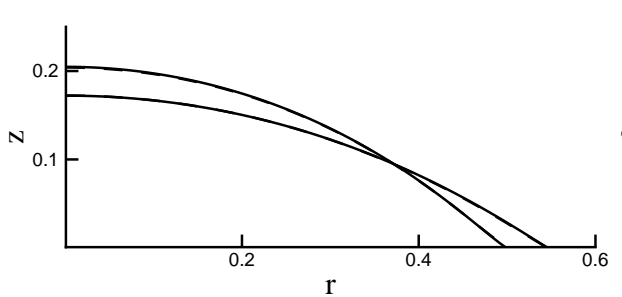

(a)

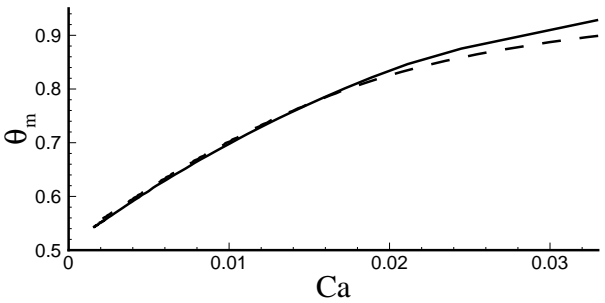

(b)

FIGURE 10. (a)Snapshots of interface shapes during spreading; (b) apparent contact angle as a function of instantaneous $C a$ at $r_{d}=r_{v}=0.1, O h=0.1, \theta_{i n i}=\pi / 3, \theta_{w}=\pi / 6$ and $\lambda=0.001$. Solid lines are results from LS method and dashed lines are from DI method.

depend on the slip length: at $\lambda=0.001$, the coefficient has reduced to 0.5 , and further down to 0.36 at $\lambda=0.0002$. Care should be taken though with using these results in flows other than those studied here. Also, in diffuse interface methods, the mobility is usually related to the interface thickness following a power law $M \sim \zeta^{n}$; herein we have used $n=2$ (as in Ding \& Spelt (2007a)), but other values of $n$ ranging from -1 to 1 have been used previously, and it is expected that a different relation between the equivalent slip length and the interface thickness would then be found.

\subsection{Dewetting}

Cox (1998) considered mainly inertial spreading, not rapid dewetting. Nevertheless, the inner region in rapid spreading is the same as in slow spreading, which in turn also applies to dewetting; and Cox (1998) argued that the result of his inviscid analysis is similar to that for slow spreading also. To put this on a firmer basis, we briefly investigate rapid dewetting here.

Before turning to inertial effects though, we first compare in Fig.11a the results for more gradual dewetting with Hocking \& Rivers (1982), which is also expected to be applicable for dewetting (note, the capillary number is defined here to be negative in dewetting). Overall, the conclusions drawn in the above on slow spreading carry over, the results of theory and simulations agree.

Rapid dewetting is studied in Fig. 11b-d. First, successive snapshots of the drop shape are shown in Fig 11b. Inertial effects are clearly seen, in that initially not the entire drop is pushed back and upwards. Detailed investigation of Fig 11b suggests a capillary wave travels back from the centre of the drop and approaches the contact line, which causes strong oscillation of the apparent angle (Fig. 11d). In Fig.11d, the apparent angle obtained from numerical simulation is compared with the theory of Cox (1998) after the modifications indicated in Sec. 4. The same parameter values $\left(R e^{*}, d_{m}\right)$ as in the analogous comparisons for spreading are used here (except $\epsilon=\lambda$ is now used). Although the theory of Cox (1998) can be used to predict the apparent angle, inspection of the results in Fig. 11c for $\theta(s)$ shows that the theory cannot be used for any further level of detail; especially a large discrepancy occurs in the inviscid intermediate sublayer, and the favourable comparison for the apparent angle seems rather fortunate.

At $O h=0.01$ when the wetting angle is sufficiently large, for example $\theta_{w}=2 \pi / 3$, the difference between the initial angle and the wetting angle triggers a large contact line velocity which can lead to a jump up of the droplet from the surface, see Fig. 12a-g, where the instantaneous interface profiles during such a process presented. The fluid initially near the contact line is rolled up and a surface wave travels to the drop centre. The wave 


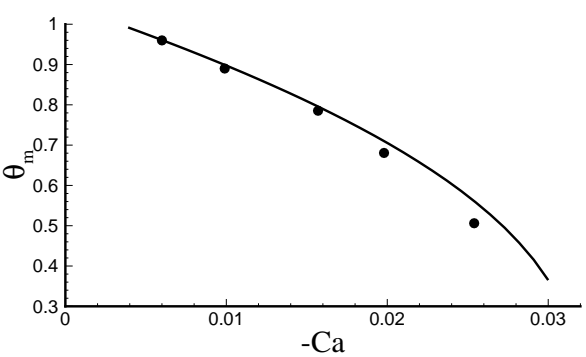

(a)

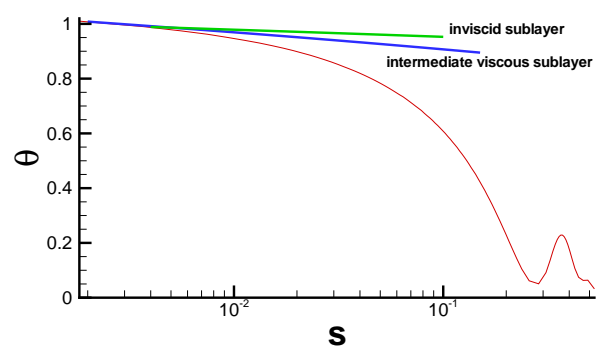

(c)

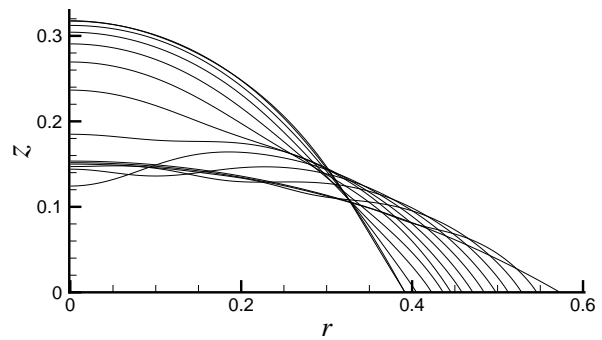

(b)

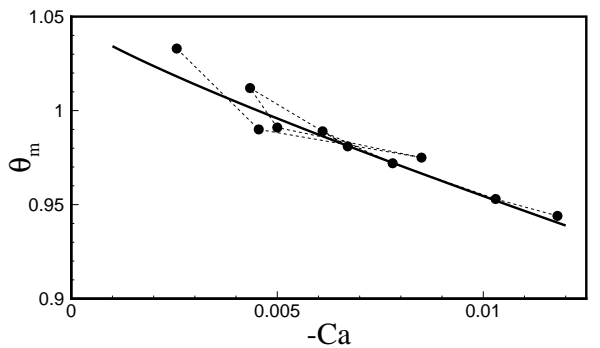

(d)

Figure 11. Results for dewetting. (a) Comparison of apparent contact angle vs $C a$ against Hocking \& Rivers (1982) (solid line) for slow dewetting for $O h=0.1$. (b)-(d) Results for the inertia-dominated regime, $O h=0.01$ : in (b), the drop shape evolution is shown, in (c), the shape is compared with Cox (1998) as modified herein, with $\epsilon=\lambda, R e^{*}=0.37$, and (d) shows the corresponding comparison for the apparent angle, where $d_{m}=0.2$ is used. Other parameters are $\theta_{\text {ini }}=\pi / 6, \theta_{w}=\pi / 3, \lambda=0.001, r_{d}=r_{v}=0.1$.

moves so fast that there is no time for the interface at $r=0$ to go up, and a pocket of air is trapped. The centre of mass of the drop goes up and before a maximum height is achieved, the contact line has arrived at the centre line and thus the whole droplet jumps up. For this complex situation, we find Cox (1998) to be only of limited use for predicting the apparent angle and the interface shape close to the contact line, as shown in Fig. $12 \mathrm{~g}$ for the comparison of the apparent angle. A further complication at large dewetting rates may expected to be an approach to a Landau-Levich-Derjaguin-type limit observed in rapid withdrawal of a plate from a pool (Landau \& Levich (1942)): beyond a critical value of $-\mathrm{Ca}$, a macroscopic film would then be expected to form. However, in the latter, the flow is driven by the moving plate with the liquid nearly passively adjusting itself to it, whereas in dewetting droplets, the moving contact line drives the flow. For rapid withdrawal of a plate from a pool, a detailed analysis and corresponding prediction of the critical value of $-C a$ are available (Eggers (2004)) but these involve the angle between the plate and the gravitational acceleration. A less advanced, but local prediction proposed by de Gennes (1986) that can be implemented here as an estimate gives a critical value $-C a^{c}=\theta_{w}^{3} /\left(9 \sqrt{3} \ln \left(\lambda^{-1}\right)\right.$ ) (where we have used $\lambda$ as the ratio of a micro- and macrolengthscales). This is -0.085 for the case studied in Fig. 12, which is above the range of values attained there. However, for the case in Fig.11, in early stage of the simulation the capillary number has reached a value of -0.1 , which is well beyond the critical capillary number of 0.01 predicted by the de Gennes formula, but we have not observed any film being formed. 

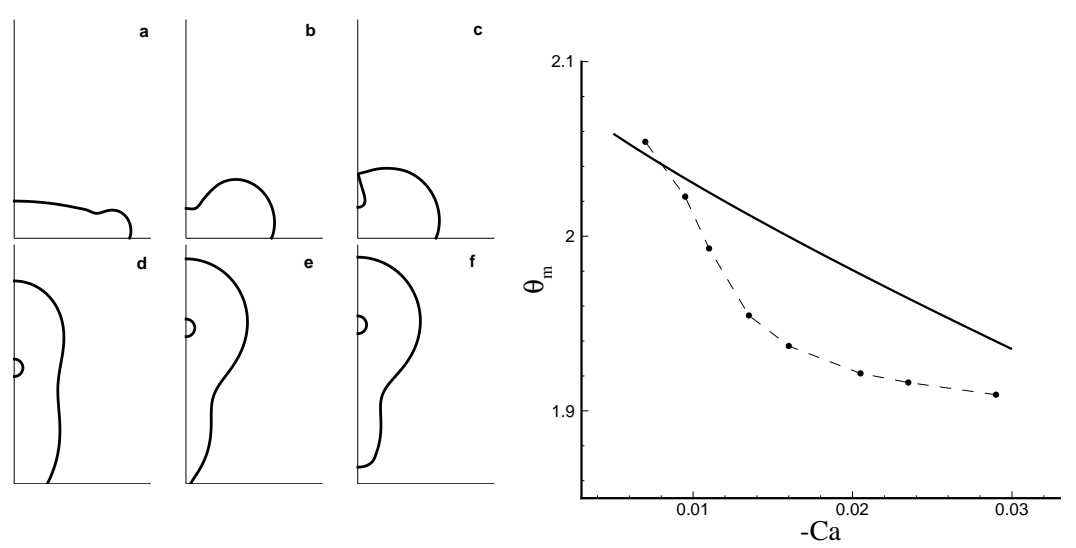

(g)

Figure 12. (a)-(f) Instantaneous interface profiles during the dewetting of a droplet at $O h=0.01$ and $\theta_{w}=2 \pi / 3$. Other parameters are same to Fig. 11. (g) Apparent angle as a function of $\mathrm{Ca}$. Dots are from numerical simulation and solid curve is from Cox (1998) with $\epsilon=\lambda, R e^{*}=0.37$ and $d_{m}=0.2$.

\subsection{Droplet impact}

We have also carried out simulations of droplet impact by giving the droplet an initial downwards velocity. The purpose is to test whether the inertial theory of Cox (1998) can still predict a reasonable apparent angle for such situations. The Weber number, defined as $W e=\rho_{1} U_{i}{ }^{2} D / \sigma$ where $U_{i}$ is the impact velocity, is employed to identify the extent of impact. Fig. 13a presents the comparison of apparent angles obtained from numerical simulation (symbols) and Cox (1998) (solid curve) for a case with $W e=5$, and it is seen that reasonably good agreement is achieved. The values of parameters used in the theory are $\epsilon=1.5 \lambda, R e^{*}=0.37, d_{m}=0.2$. With moderate impact velocity (i.e. $W e=5$ ), capillary-type waves emit from the contact line, travel upwards along the interface and reflect back, which is in general similar to rapid droplet spreading. However, at high impact speed, the impact velocity is much larger than the capillary wave (e.g., Renardy et al. (2003)) and the contact line region forms a thin layer of liquid, as illustrated in Fig. $13 \mathrm{~b}$ for $W e=33$ (the perhaps familiar phenomenon of crown splashing is only observed in impact on pre-wetted or very rough surfaces that are both beyond the scope of this study Yarin (2006)). The apparent angle is then very difficult to be extrapolated accurately. Finally, at extreme impact velocities such that $R e$ is no longer smaller than $\lambda^{-1}$ and inertial effects become significant in the inner region, the theory outlined in Sec. 4 does not hold. That regime has been investigated separately by Cox (1998); we also note that in high-speed impact, the gas is compressed rather than drained into a layer of a few tens of nanometers thick, during which the drop 'skates' on an air cushion before a contact line is formed at multiple sites, beyond the scope of the present study (Kolinski et al. (2012)).

\subsection{Comparison of adjusted analysis with experiments on rapid spreading}

Prior comparison of asymptotic analysis with experimental data has been mainly in the slow spreading regime, and in most cases for contact-line motion in capillaries or over a moving plate. A quantitative comparison yields an approximate value of an effective slip length (e.g., Marsh et al. (1993)). In some cases, the additional terms in the theory 


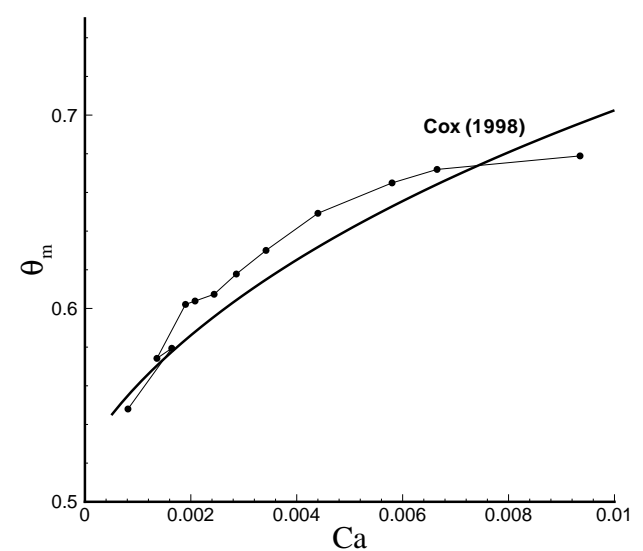

(a)

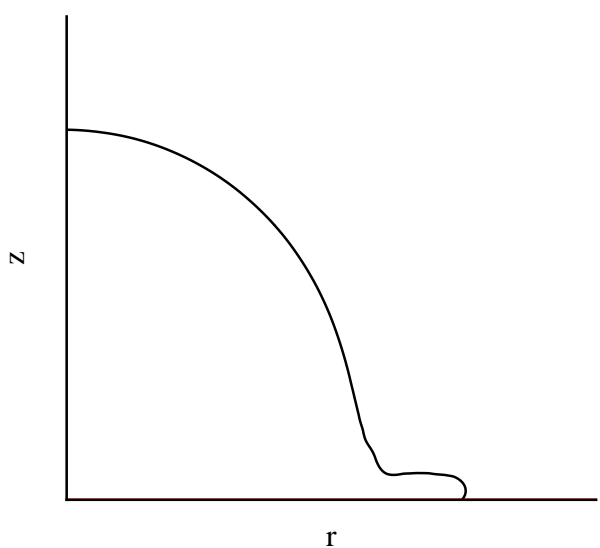

(b)

FiguRE 13. (a)Apparent angle as a function of contact line speed. $W e=5, O h=0.0022$, $\theta_{i n i}=\pi / 3, \theta_{w}=\pi / 6, r_{d}=r_{v}=0.1$ and $\lambda=0.0001$ (b) Illustration of the instantaneous interface profile at higher impact velocity $W e=33$.

such as $Q_{1}$ seem to have been ignored, which would result in an error in the inferred slip length. A direct comparison with the theory of Hocking \& Rivers (1982) for droplet spreading with experiment was conducted by those authors in their paper, and good agreement was obtained for what seems a reasonable value of the slip length (10nm). It may therefore seem surprising that for rapid spreading, although the theory for the inner region and viscous intermediate sublayer in the analysis of Cox (1998) are in effect identical to their counterparts for slow spreading, large discrepancies appear to have been found.

In Fig. 14, we reproduce the experimental data on the apparent contact angle versus instantaneous contact-line speed (made dimensionless), taken from Ding et al. (2012). The apparent angle in the experiment is measured for a $0.77 \mathrm{~mm}$ diameter water droplet (corresponding to a small Bond number, $\rho g D^{2} / \sigma=0.083$, where $g$ is the gravitational acceleration; we have confirmed numerically that there is no significant effect of gravity on the results in this case) by extrapolating the interface shape from a macroscopic length scale, of about $\mathrm{O}(50 \mu \mathrm{m})$, to the contact line and finding the intersecting angle with the wall. This is for a case wherein spreading is so rapid that a capillary wave eventually leads to pinch-off; the particular type of satellite ejection is referred to as second-stage pinch-off and is illustrated in Fig. 14(b). Unlike in that prior work, a direct comparison with the theory of Cox (1998) is now possible, following the detailed validation conducted herein and subsequent refinement of the theory. In the figure, we first show as a reference the theoretical result when using the expected values of a slip length of $1 \mathrm{~nm}$ (see Ding et al. (2012) for details of the surfaces used) and the static advancing angle of $23^{\circ}$. The apparent angle is clearly vastly underpredicted. We have first determined a fitted value of the slip length that would lead to the theory to agree well with the experiments. It is indeed possible to obtain a reasonable curve fit (the contact angle used was kept at the static angle determined experimentally), as shown in Fig. 14, but the resulting value of the slip length is unphysical, $10^{-7} \mathrm{~nm}$, throughout the entire spreading process. Although it is tempting to attribute this to a limitation of a slip model, from the recent work of 


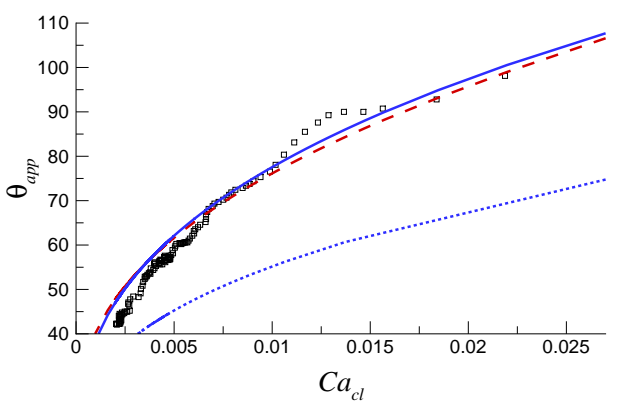

(a)

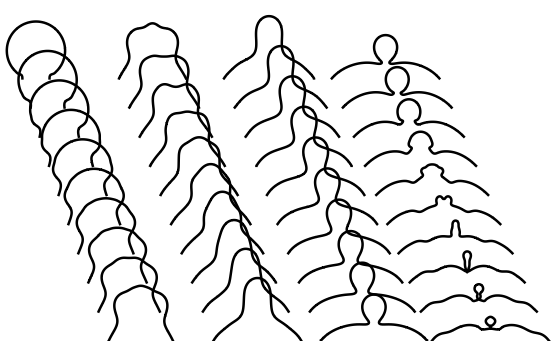

(b)

Figure 14. (a) Apparent contact angle as a function of instantaneous $\mathrm{Ca}$ for experiment of Ding et al. (2012) (symbols) and theory Cox (1998) as modified in accord with the present computational results (lines). The experimental data are for a $0.77 \mathrm{~mm}$ water droplet at $O h=0.006$ and static angle $23^{\circ}$. The dotted and solid lines are the theory for the experimental contact angle and a slip length of $1 \mathrm{~nm}$ and $10^{-16} \mathrm{~m}$, respectively. The dashed line is the theory for a slip length of $1 \mathrm{~nm}$ and a velocity-dependent contact angle.(b) Illustration of a second stage pinch off taken from Ding et al. (2012).

Savva \& Kalliadasis (2011), other models (such as a precursor film) are expected to result in a similar discrepancy.

In all the results presented so far here, however, we have kept the actual contact angle (in the inner region) fixed. Although Hocking \& Rivers (1982) found their model to agree well with their experiments for slow spreading when using a fixed slip length and contact angle, Sheng \& Zhou (1992), comparing the analysis of Cox (1986) with the experiments of Fermigier \& Jenffer (1990), only obtained close agreement when accounting for a velocity-dependent contact angle, $\theta_{d}$; Bayer \& Megaridis (2006) argued for the use of such model in their study of droplet spreading. Models of contact lines moving over defects that equate an effective drag force exerted by a defect on the contact line to the total applied force that includes a capillary forcing arising from surrounding defects (e.g., Ertas \& Kardar (1994)) do predict that the dynamic contact angle (on the scale of roughness elements, not to be confused with the present apparent angle) increases with the contact-line speed, and the contact line to advance in jumps or avalanches as it depins from defects. There is experimental evidence of avalanche-type behaviour (David \& Neumann (2010)). As reviewed in Sheng \& Zhou (1992), several forms have been proposed for such a relation between contact angle (in the inner region) and the contact-line speed. In fact, even for contact line motion over perfectly smooth surface, molecular dynamics simulations by Qian et al. (2003) and Ren \& E (2007) have shown that the contact angle deviates from the equilibrium static angle and the deviation is proportional to the contact line speed. It is not attempted here to investigate the detailed physics in the inner region or argue for a specific proposal of the contact angle and the contact line speed relation for rapid spreading (especially since all prior theoretical work in this area is for slow spreading, to our knowledge), but it is important to investigate whether at least a modest change in the contact angle with contact-line speed can explain the large gap between the theory of Cox (1998) and the experimental data in Fig. 14, or if other physics must be accounted for. We have therefore included in this figure the theoretical prediction based on a contact-angle/speed relation of the form proposed by Sheng \& Zhou (1992), i.e., $\cos \theta_{w}=\cos \theta_{d}+B \cdot C a^{n}$. The result shown is for fitted values $B=9.6, n=0.64$, which are both of the same order as the values inferred by Sheng \& Zhou (1992) (they found different prefactors for different viscosity ratios and different 
values of $\theta_{w}$ ). Note, we have kept the static angle at the experimental value although it appears to be a slight overestimate, the difference with a fit using $n<0.45$ could be of the same order of magnitude as that caused by the uncertainty in the value of $\theta_{w}$.

In conclusion, the results of the validation and refinement theory of Cox (1998) are not inconsistent with experimental data on rapid droplet spreading, if one allows for the contact angle to increase modestly with contact-line speed. Although introduction of a velocity-dependent microscale contact angle is unsatisfactory in that this introduces yet further parameters in the model, the fact that values found previously for slow spreading of these coefficients can bridge the large gap in Fig. 14 between the theory for a fixed static angle and experimental data that could otherwise only be achieved by reducing the slip length by seven orders of magnitude, is compiling.

We have not been able to undertake a comparison with the experimental data of Bayer \& Megaridis (2006), although these are also for rapid droplet spreading. This is mainly because the accurate value of the static advancing angle, required in Cox (1998), is not provided in the experiment, which introduces further uncertainty in an inferred value of $\lambda$. Indeed, a comparison was not presented by Bayer \& Megaridis (2006). The main visible trend in their data is that at large contact-line velocity, the apparent angle depends on the impact velocity of the drop. In Sec. 5.5, such dependency has not been observed, and our results are still in reasonable agreement with the theory of Cox (1998) even at the impact speeds used by Bayer \& Megaridis (2006). Evidently, further experimental work in this area would be highly desirable.

\section{Conclusions}

Asymptotic analyses for slow and rapid spreading of droplets have been tested and, in places, modified, using a newly developed computational method, a slip-length based level set method integrated in the efficient adaptive mesh refinement tool package PARAMESH, for dimensionless slip length values down to $10^{-4}$. For slow spreading, the results in Sec. 3 are in very close agreement with the theory of Hocking \& Rivers (1982), for situations where $C a$ is less than $O(0.01)$ and $\lambda$ smaller than $O(0.001)$, although it was found that the theory was fortuitous in matching the outer region to an intermediate region at the apparent angle, whereas the apparent angle is not reached anywhere on the surface. A simple extension of the model of Hocking \& Rivers (1982) to account for finite viscosity of the outer fluid has been shown to represent the data well. The flow pattern near the contact line has been shown to be consistent with that in e.g. Cox (1986), with the separating ejection streamline lying in the less-viscous fluid, and a wedge-type pattern in the more viscous fluid. Furthermore, the wall shear stress and pressure in rapid spreading are in excellent agreement with the theory. We have supplemented the theory by presenting an explicit relation for the pressure in the inner region based on the numerics.

For fast spreading, in Sec. 4 we have assessed in detail the use of the theory of Cox (1998) for the practically useful case of $1 \ll R e \ll \lambda^{-1}$, where $R e$ is the Reynolds number based on the contact-line speed, and $\lambda$ is the dimensionless slip length. As is, this theory was not found to lead to an accurate prediction of the simulation results, and it was established that this is due to ignored higher-order terms (corrected to $\mathrm{Ca}^{+1}$ ) and also the values of parameters $\left(\epsilon, R e^{*}\right)$ that should be used in the theory. Most of these have been estimated by comparing the detailed shape from the numerics with the theory. A further difficulty was an ambiguity in the definition of the apparent contact angle. We have proposed to insist on the same definition as used in theory for slow spreading, i.e., the value obtained by extrapolating from the outer region; we have also shown that using the maximum angle long the interface, although more convenient (and 
used in our earlier work, Ding \& Spelt (2007a)), leads to inconsistent results, especially at early times, when a large-amplitude capillary wave travels from the contact line to the top of the drop. In the end, the theory, thus modified, agreed well with the simulations, again for $C a<O(0.01)$ and $\lambda<O(0.001)$. Further, the results indicate that the wall shear stress and pressure in the inner region are close to the corresponding results for slow spreading, consistent with the notion that in that region, the theories for slow and rapid spreading coincide. Although the theory of Cox (1998) was not derived for rapid dewetting, we have demonstrated that it can be used to represent the apparent angle vs $\mathrm{Ca}$ reasonably well at moderate dewetting speeds, although the theory fails to predict the shape of the drop accurately, but that the use of the theory is further reduced in extreme cases of dewetting.

We have also found that the modified theory of Cox (1998) can be used to predict to a reasonable degree of approximation the apparent angle for rapid droplet spreading with moderate initial impact. It may be noticed that we have three additional coefficients in our modification of the theory of Cox (1998). These have clear physical meanings and have been determined separately from different parts of the detailed shape of the interface, and are not just fitting coefficients. If, in practice, one would want to only know the apparent angle and combine these coefficients, this is possible, as follows (we consider here only the case $\left.r_{v}=0\right)$. The theoretical apparent angle was obtained from (4.7), with (4.2) to give $\theta^{*}$, where $g_{i v}$ is defined by (4.4), $h_{i v}$ by (4.5) and $g$ by (3.6). This can be simplified by using Taylor series for the integrand in (3.6) and for $g_{i v}$, only keeping the first term. The result can be written as

$$
C a^{-1}\left(\theta_{m}^{3}-\theta_{w}^{3}\right)=\frac{6}{1.53161} \ln R e+9 \ln \left((\lambda R e)^{-1}\right)+Q_{2}\left(\theta_{w}\right)
$$

with a sole parameter defined as

$$
Q_{2}\left(\theta_{w}\right) \equiv \frac{6}{1.53161} \ln \left(d_{m} / R e^{*}\right)+9 \ln \left(R e^{*} \lambda / \epsilon\right)+9 Q_{i} f\left(\theta_{w}\right)+9\left[h_{i v}\left(\theta_{m}\right)-h_{i v}\left(\theta^{*}\right)\right] .
$$

The simulations indicate $d_{m} \approx 0.2, \epsilon=1.5 \lambda$ and $R e^{*} \approx 0.37\left(Q_{i} f=1+Q_{1}\right.$, and $Q_{1}$ is documented by Hocking \& Rivers (1982)).

The above simplified form also gives us an opportunity to easily assess the significance of the additional terms, as these are represented by $Q_{2}$. We have already demonstrated that for $\lambda=10^{-4}$, ignoring the additional term leads to a significant error (cf. Fig. 6); for a millimeter-sized droplet, this corresponds to a slip length of $100 \mathrm{~nm}$. At the low-end of the range of expected slip length values $(1 \mathrm{~nm})$, the above expression gives a discrepancy of about half that seen in Fig. 6.

The present findings open up the possibility of future work on large-scale computations involving rapid spreading phenomena wherein such a relation between apparent angle and contact-line speed is required, and a slow-spreading analysis cannot be used, except for the wall shear stress and pressure. A concern is, however, that the microscale contact angle may have to be taken to depend on the contact-line speed (see Sec. 5.6), which introduces further empirical parameters. The present work also shows the need for further experimental study of especially the contact-line dynamics in impact of droplets on a dry surface.

The authors would like to acknowledge financial support from EPSRC under grant numbers EP/E046029/1. The simulations were performed using the high performance computer clusters provided by the Imperial College London, Queen Mary University of London and those of CINES/Jade (c2011026679,c2012026893). The authors are grateful for useful discussions with Dr F.H. Zhang and Dr E.Q. Li about the experimental mea- 


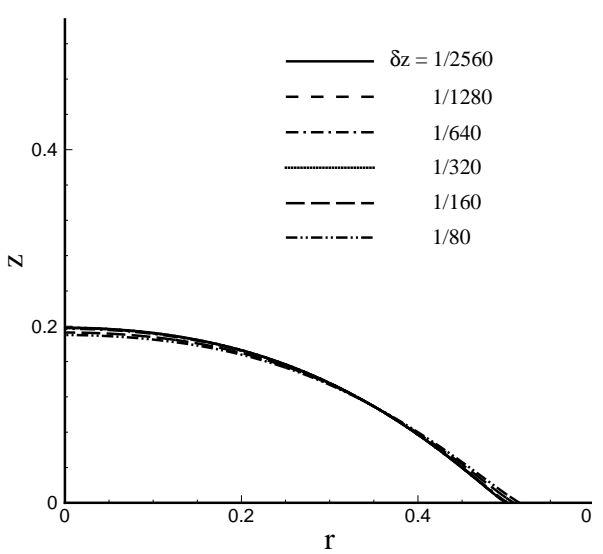

(a)

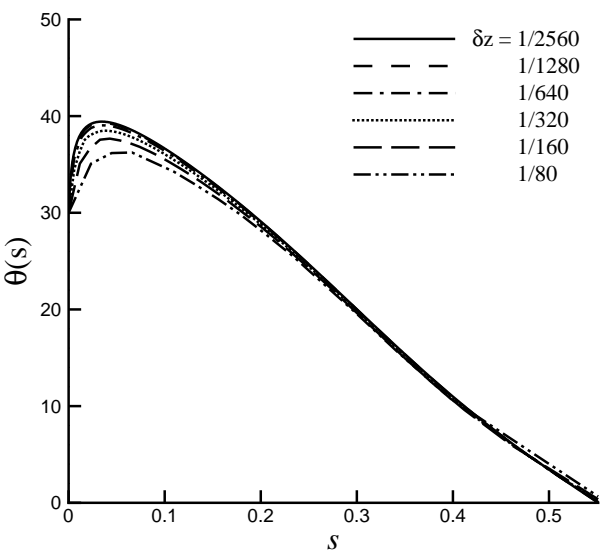

(b)

FIGURE 15. (a) Instantaneous interface shapes with different mesh resolutions for $r_{d}=r_{v}=0.1$, $\lambda=0.001, O h=0.0316, \theta_{i n i}=\pi / 3, \theta_{w}=\pi / 6$ (b) angle that the interface makes with the wall as a function of arc length to the contact line.

surement of contact angles. The authors also want to thank Dr H. Ding for his helpful comments on the revision of the manuscript.

\section{Appendix: Grid convergence and singularity study}

We investigate the extent to which asymptotic behaviour expected from prior analytical work is confirmed by the present numerical simulation.

The shape of the interface is found to converge if the grid spacing approaches the slip length, see Fig.15 for results at $\lambda=0.001$ (reasonably good convergence appears to have been reached at a grid spacing of $\delta z=1 / 320$ in this case). We analyze the converged results in detail in Sec. 3 and 4.

The corresponding instantaneous shear rate and pressure along the wall with slip are presented in Fig.16. The peak value of the shear rate appears to converge, although a fully-converged value is not yet obtained at $\delta z=1 / 2560$, albeit that the interface shape has converged at a much larger grid spacing (Fig.15). By contrast, the peak value of pressure at the contact line exhibits no evidence of convergence. These results are in fact consistent with results from lubrication theory (e.g., Devauchelle et al. (2007)), as can be seen in Fig.17: the pressure around the contact still diverges as $\ln \left(\left|r_{c}-r\right|\right)$ (the results shown there are for a larger slip length in order to allow inclusion of simulations wherein the grid spacing is very much smaller than the slip length; corresponding results for the same case as in Fig.16 are consistent with those shown in Fig.17, but naturally do not extend to such extreme resolutions). In conclusion, slip regularizes the singularity in wall stress but an integrable singularity in the pressure remains.

Throughout the paper, results for a dimensionless slip length ranging from $10^{-2}$ to $10^{-4}$ are presented and discussed. Grid dependence studies for each slip length have shown that when the mesh size approaches the slip length, $\delta z / \lambda<2$, results for the interface shape and contact line spreading rate have converged; using somewhat larger grid spacing results in a minor difference. In the following, results are presented for grid spacings of $\delta z=1 / 320,1 / 640$ for a slip length of $10^{-2}, 10^{-3}$, respectively. In sections 3.1 and 3.2 for $\lambda=10^{-4}, \delta z=1 / 5120$ has been used. Since the results were found to differ 


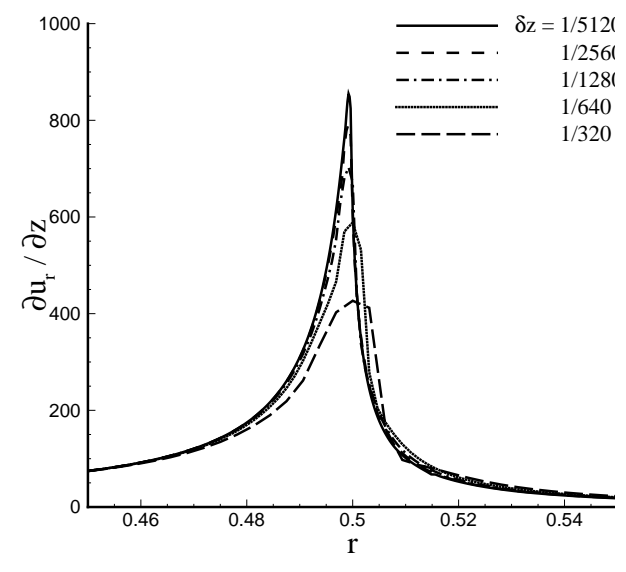

(a)

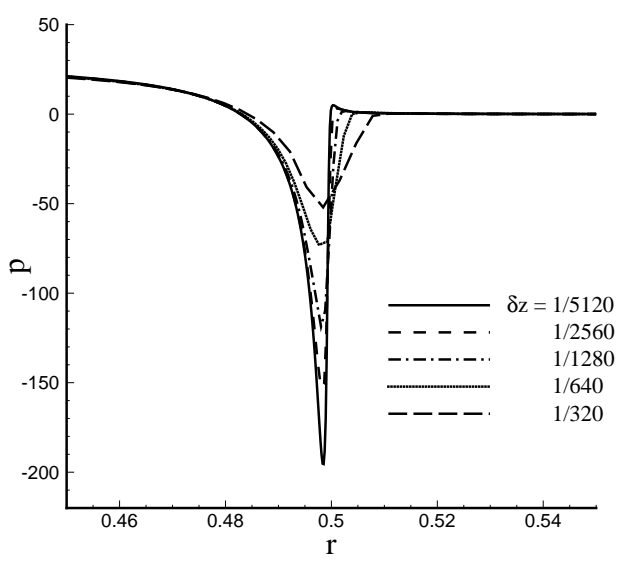

(b)

FigURE 16. Instantaneous (a) shear rate and (b) pressure along the wall with different mesh resolutions for $r_{d}=r_{v}=0.1, \lambda=0.001, O h=0.0316, \theta_{i n i}=\pi / 3, \theta_{w}=\pi / 6$.

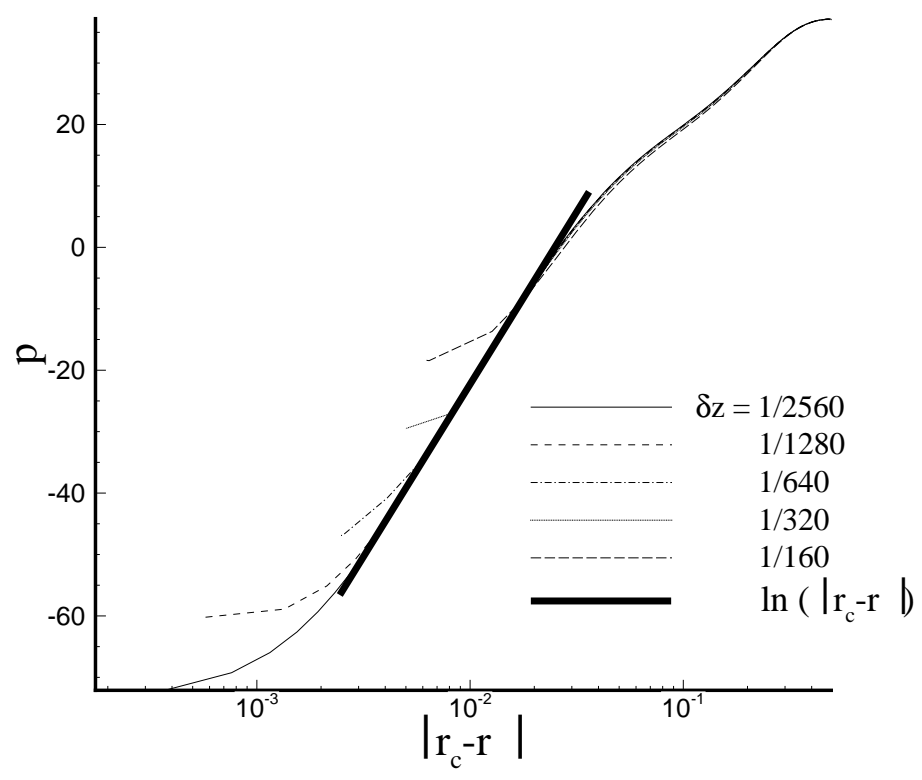

FiguRE 17. Pressure as a function of distance to the contact line with different mesh resolutions for $r_{d}=r_{v}=0.1, \lambda=0.01, O h=0.0316, C a=0.019, \theta_{i n i}=\pi / 3, \theta_{w}=\pi / 6$.

only by less than $2 \%$ from those obtained with $\delta z=1 / 2560$, this latter value has been used in the remaining sections for $\lambda=10^{-4}$. The accuracy of the results on these grids is further tested in the following by detailed comparison against theoretical results, where available. A typical simulation using 64 processors (Fortran MPI), with $\delta z=1 / 2560$ used around 50,000 CPU hours; a run with $\delta z=1 / 5120$ took almost 140,000 hours. 


\section{REFERENCES}

Afkhami, S., Zaleski, S. \& Bussmann, M. 2009 A mesh-dependent model for applying dynamic contact angles to VOF simulations J. Comput. Phys. 228, 5370-5389.

BAYER, I. S., AND Megaridis, C. M. 2006 Contact angle dynamics in droplets impacting on flat surfaces with different wetting characteristics. J. Fluid Mech. 558, 415-449.

BAzhlekov, I. B., AND Chesters, A. K. 1996 Numerical investigation of the dynamic influence of the contact line region on the macroscopic meniscus shape. J. Fluid Mech. 329, 137-146.

Bonn, D., Eggers, J., Indekeu, J., Meunier, J. And Rolley, E. 2009 Wetting and spreading. Rev. Mod. Phys. 81, 739-805.

Bussmann, M., Mostaghimi, J. \& Chandra, S. 1999 On a three-dimensional volume tracking model of droplet impact. Phys. Fluids 11, 1406-1417.

Chen, Q., Ramé, E. And Garoff, S. 1997 The velocity field near moving contact lines. J. Fluid Mech. 337, 49-66.

Christodoulou, K.N. \& Scriven, L.E. 1992 Discretization of free surface flows and other moving boundary problems. J. Comput. Phys. 99, 39-55.

Cox, R. G. 1986 The dynamics of the spreading of liquids on a solid surface. Part 1. Viscous flow. J. Fluid Mech. 168, 169.

Cox, R. G. 1998 Inertial and viscous effects on dynamic contact angles. J. Fluid Mech. 357, $249-278$.

David, R. \& Neumann, A.W. 2010 Computation of contact lines on randomly heterogeneous surfaces. Langmuir 26, 13256-13262.

De Gennes, P. G. 1986 Deposition of Langmuir-Blodgett layers. Colloid Polym. Sci. 264, 463-465.

Devauchelle, o., Josserand, C., \& Zaleski, S. 2007 Forced dewetting on porous media. J. Fluid Mech. 574, 343-364.

Ding, H. \& Spelt, P. D. M. 2007a Inertial effects in droplet spreading: a comparison between diffuse interface and level-set simulations J. Fluid Mech. 576, 287-296.

Ding, H., Spelt, P. D. M. \& Shu, C. 2007b Diffuse interface model for incompressible twophase flows with large density ratios J. Comput. Phys. 226, 2078-2095.

Ding, H. \& Spelt, P. D. M. 2008 Onset of motion of a 3D droplet on a wall in shear flow at moderate Reynolds numbers. J. Fluid Mech. 599, 341-362.

Ding, H., Gilani, M.N.H. \& Spelt, P. D. M. 2010 Sliding, pinchoff and detachment of a droplet on a wall in shear flow. J. Fluid Mech. 644, 217-244.

Ding, H., Li, E.Q., Zhang, F. H., Sui, Y., Spelt, P. D. M. \& Thoroddsen, S. T. 2011 Ejection of small droplets in rapid drop spreading. J. Fluid Mech. 697, 92-114.

Dussan V., E. B. \& Davis, S. H. 1974 On the motion of a fluidfluid interface along a solid surface. J. Fluid Mech. 65, 71-95.

Eggers, J. 2004 Hydrodynamic theory of forced dewetting. Phys. Rev. Lett. 93, 094502.

Eggers, J. \& Stone, H. A. 2004 Characteristic lengths at moving contact lines for a perfectly wetting fluid: the influence of speed on the dynamic contact angle. J. Fluid Mech. 505, 309-321.

Ertas, D. \& Kardar, M. 1994 Critical dynamics of contact line depinning. Phys. Rev. E 49, R2532-R2535.

Fermigier, M. \& Jenffer, P. 1990 An experimental investigation of the dynamic contact angle in liquid-liquid systems. J. Colloid. Interf. Sci. 146, 226-241.

Foister, R. T. 1990 The kinetics of displacement wetting in liquid/liquid/solid systems. J. Colloid. Interf. Sci. 136, 266-282.

Fuentes, J. \& Cerro, R. L. 2005 Flow patterns and interfacial velocities near a moving contact line. Exp Fluids 38, 505-510.

Fukai, J., Shilba, Y., Yamamoto, T., Miyatake, O., Poulikakos, D., Megaridis, C.M. \& ZHAO, Z. 1995 Wetting effects on the spreading of a liquid droplet colliding with a flat surface: experiment and modeling. Phys. Fluids 7, 236-247.

Greenspan, H.P. 1977 On the motion of a small viscous droplet th8t wets a surface. J. Fluid Mech. 84, 125-143.

Hocking, L. M. 1977 A moving fluid interface. Part2. The removal of the force singularity by a slip flow. J. Fluid Mech. 79, 209-229. 
Hocking, L. M. \& Rivers, A. D. 1982 The spreading of a drop by capillary action. J. Fluid Mech. 121,425-442.

Hocking, L. M. 1983 The spreading of a thin drop by gravity and capillarity. Q. J. Mech. Appl. Maths 36, 55-69.

Huh, C. \& Mason, S.G. 1977 The steady movement of a liquid meniscus in a capillary tube. J. Fluid Mech. 81, 401-419.

Huh, C. \& SCRIVEn, L. E. 1971 Hydrodynamic model of steady movement of a solid/liquid/fluid contact line. J. Colloid. Interf. Sci. 35, 85-101.

JACQMin, D. 2000 Contact-line dynamics of a diffuse fluid interface. J. Fluid Mech. 402, 57-88.

JiAnG, X. \& JAMES, A.J. 2007 Numerical simulation of the head-on collision of two equal-sized drops with van der Waals forces. J. Engng Math. 59, 99-121.

KAFKA, F. Y. \& DUSSAN V., E. B. 1980 On the interpretation of dynamic contact angles in capillaries. J. Fluid Mech. 95, 539-565.

Kolinski, J. M., Rubinstein, S. M., Mandre, S., Brenner, M. P., Weitz, D. A. \& MaHADEVAN, L. 2012 Skating on a film of air: drops impacting on a surface. Phys. Rev. Lett. 108, 074503.

Landau, L. D. \& Levich, B. V. 1942 Dragging of a liquid by a moving plate. Acta Physicochim. URSS 17, 42-54.

Le Grand, N., Daerr, A. \& Limat, L. 2005 Shape and motion of drops sliding down an inclined plane. J. Fluid Mech. 541, 293-315.

Liu, X. D., Osher, S. \& Chan, T. 1994 Weighted essentially non-oscillatory schemes. J. Comput. Phys. 115, 200-212.

LOWNDES, J. 1980 The numerical simulation of the steady movement of a fluid meniscus in a capillary tube. J. Fluid Mech. 101, 631-646.

MacNeice, P., Olson, K. M., Mobarry, C., DeFainchtein, R. \& Packer, C. 2000 PARAMESH: a parallel adaptive mesh refinement community toolkit. Comput. Phys. Commun. 126, 330-354.

Marsh, J. A., Garoff, S. \& Dussan V., E. B. 1993 Dynamic contact angles and hydrodynamics near a moving contact line. Phys. Rev. Lett. 70, 2778-2781.

Moffatt, H.K. 1964 Viscous and resistive eddies near a sharp corner. J. Fluid Mech. 18, 1-18.

Pismen, L.M. \& Pomeau 2000 Disjoining potential and spreading of thin liquid layers in the diffuse-interface model coupled to hydrodynamics. Phys. Rev. E 62, 2480-2492.

Qian, T., Wang, X.- P., \& Sheng, P. 2003 Molecular scale contact line hydrodynamics of immiscible flows. Phys. Rev. E 564, 333-360.

Qian, T., Wang, X.- P., \& Sheng, P. 2006 A variational approach to moving contact line hydrodynamics. J. Fluid Mech. 564, 333-360.

REN, W. AND E, W. 2007 Boundary conditions for the moving contact line problem. Phys. Fluids 68, 016306.

Renardy, M., Renardy, Y. \& LI, J. 2001 Numerical simulation of moving contact line problems using a volume-of-fluid method. J. Comput. Phys. 171, 243-263.

Renardy, Y., Popinet, S., Duchemin, L., Renardy, M., Zaleski, S., Josserand, C., Drumright-Clarke, M. A., Richard, D., Clanet, C. And Quéré 2003 Pyramidal and toroidal water drops after impact on a solid surface. J. Fluid Mech. 484, 69-83.

Savelski, M. J., Shetty, S. A., Kolb, W. B. \& Cerro, R. L. 1995 Flow patterns associated with the steady movement of a solid/liquid/fluid contact line.. J. Colloid. Interf. Sci. 176, $117-127$.

Savva, N. \& Kalliadasis, S. 2011 Dynamics of moving contact lines: a comparison between slip and precursor film models. Eur. Phys. Lett. 94, 64004.

Sheng, P. And Zhou, M. 1992 Immiscible-fluid displacement: contact-line dynamics and the velocity-depndent capillary pressure. Phys. Rev. A 45, 5694-5708.

Shikhmurzaev, Y. D. 1993 The moving contact line on a smooth solid surface. Int. J. Multiph. Flow 19, 589-610.

Sibley, D.N., Savva, N. \& Kalliadasis, S. 2012 Slip or no slip? A methodical examination of the interface formation model using two-dimensional droplet spreading on a horizontal planar substrate as a prototype system. Phys. Fluids 24, 082105.

Spelt, P. D. M. 2005 A level-set approach for simulations of flows with multiple moving contact lines with hysteresis. J. Comput. Phys. 207, 389-404. 
Spelt, P. D. M. 2006 Shear flow past two-dimensional droplets pinned or moving on an adhering channel wall at moderate Reynolds numbers: a numerical study. J. Fluid Mech. 561, 439463.

Stoev, K., Ramé, E. And Garoff, S. 1999 Effects of inertia on the hydrodynamics near moving contact lines. Phys. Fluids 11, 3209-3216.

Sussman, M., Almgren, A. S., Bell, J. B., Colella, P., Howell, L. H. \& Welcome, M. L. 1999 An adaptive level set approach for incompressible two-phase flows. J. Comput. Phys. 148, 81-124.

TAYLOR, G.I. 1962 On scraping viscous fluid from a plane surface. In: Miszellaneen der Angewandten Mechanik (Festschrift Walter Tollmien), M. Schäffer (Ed.), Akademie-Verlag, Berlin, p.313-315. See also The Scientific Papers of G. I. Taylor, G.K.Batchelor (Ed.), vol.IV, 410-413.

YARIN, A. L. 2006 Drop impact dynamics: splashing, spreading, receding, bouncing .... Annual Rev. Fluid Mech. 38, 159-192.

Yue, P., Zhou, C. \& Feng, J. J. 2010 Sharp-interface limit of the CahnHilliard model for moving contact lines. J. Fluid Mech. 645, 279-294. 